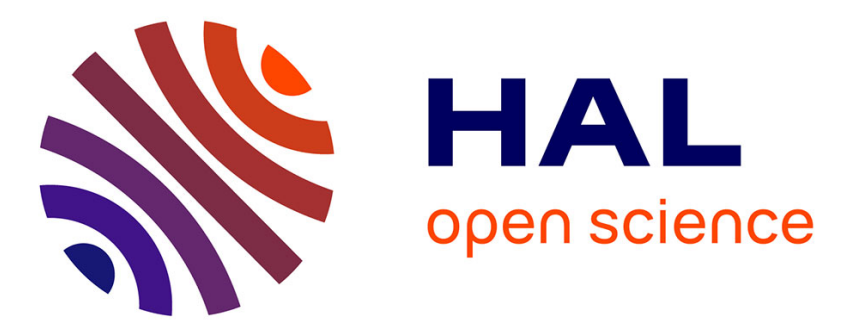

\title{
Approvisionnement et circulation du cuivre et de ses éléments d'alliage en Provence du XIIIe au XVIe siècles
}

Olivier Thuaudet

\section{To cite this version:}

Olivier Thuaudet. Approvisionnement et circulation du cuivre et de ses éléments d'alliage en Provence du XIIIe au XVIe siècles: L'apport des sources écrites et archéologiques. Nicolas Minvielle Larousse; Marie-Christine Bailly-Maître; Giovanna Bianchi. Les métaux précieux en Méditerranée médiévale: Exploitations, transformations, circulations. Actes du colloque International d'Aix-en-Provence des 6, 7 et 8 octobre 2016, Presses Universitaires de Provence; Centre Camille Jullian, pp.301-318, 2019, Bibliothèque d'archéologie méditerranéenne et africaine, 979-10-320-0230-8. hal-02306913

\section{HAL Id: hal-02306913 https://hal.science/hal-02306913}

Submitted on 7 Oct 2019

HAL is a multi-disciplinary open access archive for the deposit and dissemination of scientific research documents, whether they are published or not. The documents may come from teaching and research institutions in France or abroad, or from public or private research centers.
L'archive ouverte pluridisciplinaire HAL, est destinée au dépôt et à la diffusion de documents scientifiques de niveau recherche, publiés ou non, émanant des établissements d'enseignement et de recherche français ou étrangers, des laboratoires publics ou privés. 


\section{Les métaux précieux \\ en Méditerranée médiévale}

Exploitations, transformations, circulations

sous la direction de Nicolas Minvielle Larousse, Marie-Christine Bailly-Maître et Giovanna Bianchi

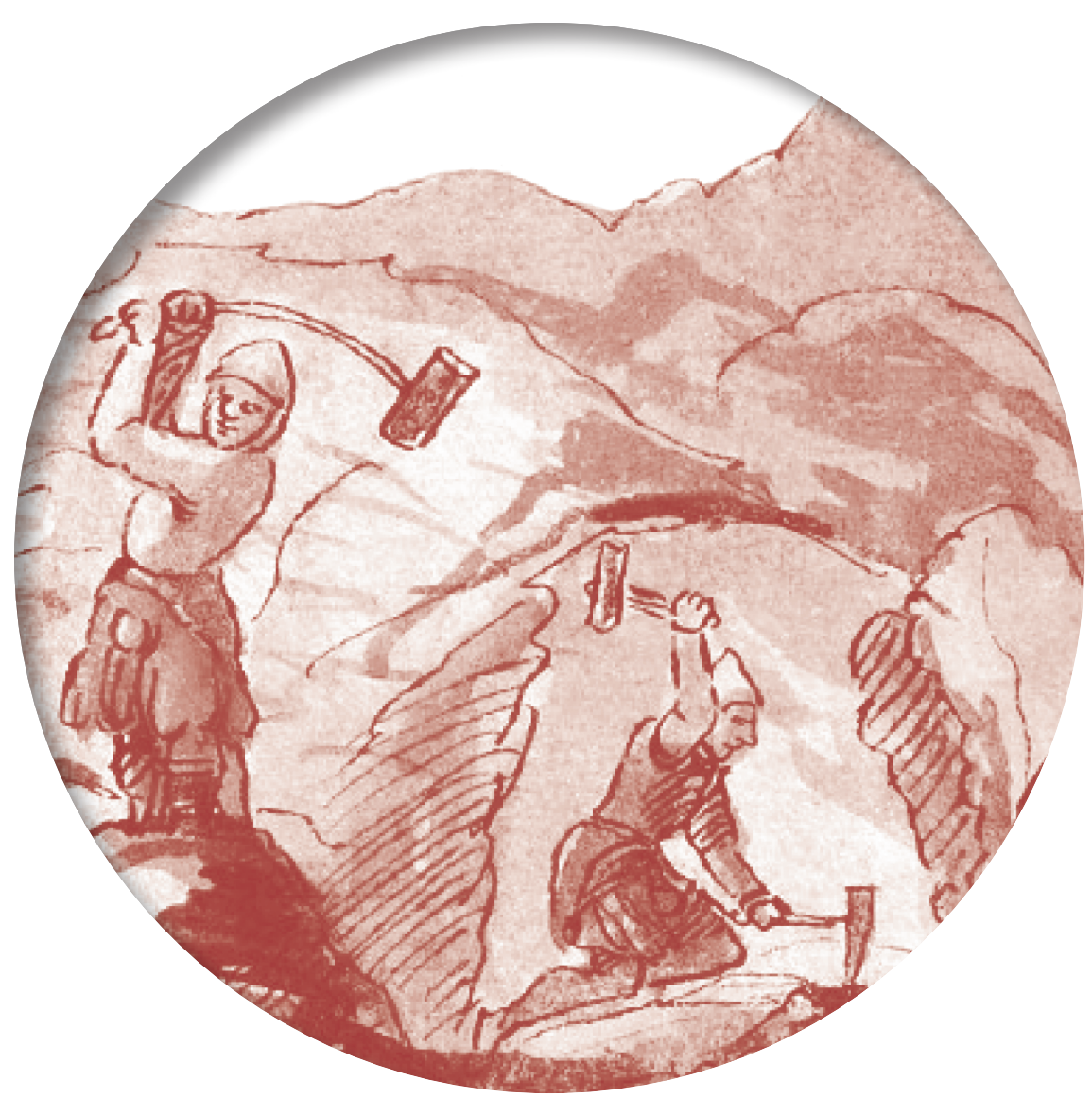


Bibliothèque

d'Archéologie

Méditerranéenne

et Africaine

\section{Les métaux précieux en Méditerranée médiévale}

\section{Exploitations, transformations, circulations}

Actes du colloque International d'Aix-en-Provence des 6, 7 et 8 octobre 2016 édités par :

Nicolas Minvielle Larousse, Marie-Christine Bailly-Maître et Giovanna Bianchi 
(c) Presses Universitaires de Provence Aix-Marseille Université

29, avenue Robert-Schuman - F - 13621 Aix-en-Provence CEDEX 1 Tél. 33 (0)4 13553191

pup@univ-amu.fr - Catalogue complet sur http://presses-universitaires.univ-amu/editeur/pup.fr

DIFFUSION LIBRAIRIES: AFPU DIFFUSION - DISTRIBUTION SODIS 


\section{Approvisionnement et circulation du cuivre et de ses éléments d'alliage en Provence du XIII ${ }^{\mathrm{e}}$ au XVle siècles}

L'apport des sources écrites et archéologiques

Olivier Thuaudet

\section{Résumé}

La restitution du circuit économique des métaux en Provence n'a jusqu'à présent pas fait l'objet de recherches poussées. Or, cet espace géographique qui ouvre sur la Méditerranée est aussi une voie de passage entre le nord de l'Europe, le midi de la France et l'Italie. Un trafic des métaux assez intense s'y est probablement déroulé.

Plusieurs sources ont été mises à contribution, en tenant compte de leurs biais propres, pour proposer une restitution de la circulation des métaux, de leur importation, de leur exportation, des acteurs économiques et des principaux centres d'utilisation de ces matériaux en Provence au cours du temps. La bibliographie régionale et extrarégionale a été dépouillée, des recherches en archives effectuées, notamment concernant l'activité minière, la localisation des martinets et les tarifs de péage.

\section{Abstract}

No advanced research has been done up to now on the economic circuit of metals in Provence. Yet, this geographical area which opens onto the Mediterranean is also a passageway between Northern Europe, the South of France, and Italy. Relatively intense metal trading is likely to have taken place there.

Several sources were called upon to make a contribution, according to their biases, to propose an account of metal circulation, importing and exporting, economic actors, and the main centers of use of these materials in Provence over time. The regional and extra-regional literature reviews have been done, and the archival research has been done, particularly on mining activity, the localization of hammers and toll rates. 


\section{Introduction}

Le cuivre et ses alliages ont été abondamment utilisés à la fin du Moyen Âge et au début de l'Époque moderne en Provence ainsi que l'illustre le mobilier mis au jour par les fouilles archéologiques. La très grande majorité de ces objets, pour certains produits dans des quantités considérables et fréquemment renouvelés tels les accessoires du costume, ne pèsent que quelques décigrammes ou grammes, fort peu donc en comparaison de produits comme les chaudrons quelques kilos - les cloches et, à la fin de la période, les pièces d'armement, dont le poids peut atteindre plusieurs centaines de kilos, mais dont la durée de vie est généralement très longue au besoin par le moyen de la réparation.

Pour la période étudiée, l'artisanat du cuivre et de ses alliages, à l'exception de quelques déchets isolés, n'est documenté en contexte archéologique que par des structures de fonte de cloche. Toutefois, les sources écrites nous informent de l'existence de fondeurs, de peyroliers - des chaudronniers - exerçant parfois aussi le métier de potier d'étain ou de martinarii, c'est-à-dire d'officiant d'un martinet à cuivre. Quelques campaniers et bombardiers se rencontrent également, un même artisan pouvant être qualifié des deux termes. En outre, l'étude de la répartition géographique et chronologique atteste l'existence de productions spécifiques à la Provence, par exemple des accessoires du costume ${ }^{1}$. L'ampleur de l'activité de transformation du cuivre et de ses alliages reste cependant difficile à évaluer. Des éléments de réponse peuvent être apportés par une recherche de l'origine des matériaux mis en œuvre: le cuivre, l'étain, la calamine et le plomb.

Les métaux sont des produits qui peuvent voyager sur de longues distances, car la plupart des territoires ne possèdent pas les ressources métalliques nécessaires à leurs propres besoins, ou en quantités insuffisantes. Comprendre les modalités d'approvisionnement et de circulation des matières métalliques en Provence, voie de passage entre le nord de l'Europe et les pays du pourtour méditerranéen, entre la Péninsule ibérique et l'Italie, nécessite d'identifier les zones d'exploitation, qui peuvent être parfois lointaines, et donc d'élargir le champ géographique d'investigation en identifiant les zones d'exploitation. Il n'existe actuellement pas de synthèse sur l'exploitation minière et le commerce des métaux dans le sud-est de la France et les informations sont donc très dispersées. Un important dépouillement bibliographique - documentation scientifique, descriptions anciennes par des voyageurs ou par des érudits provençaux - a été complété de recherches ciblées dans les archives. Les données présentées n'ont pas été rassemblées avec un objectif d'exhaustivité, mais afin de donner un aperçu relativement

1 Thuaudet 2015, 1390-1391. avancé du sujet. Nous n'aborderons pas faute de place les demi-produits tels que les fils, les feuilles métalliques et les " papiers » pour dorer, argenter ou étamer.

\section{Quelles sources d'archives?}

Trois groupes principaux de documents d'archives sont susceptibles d'être exploités dans le cadre de la thématique de la circulation des métaux. Un premier groupe est constitué d'actes de vente devant notaire, de comptes de constructions, de livres de comptes de marchands, de comptes de dépenses de la chambre apostolique d'Avignon. Cependant, ces documents, notamment les actes de vente, sont difficiles à trouver dans la masse des archives notariales et les renseignements utiles sont finalement peu nombreux.

On peut également compter sur les comptes journaliers de péage ou les comptes des entrées et sorties portuaires. Certaines de ces sources ont toutefois le défaut d'être généralement ponctuelles, soumises aux aléas des évènements politiques, économiques et sanitaires et les informations qu'elles contiennent sont pour le moins inégales. Ainsi, la nature des marchandises est loin d'être toujours spécifiée. Les données des comptes de péage sont également biaisées par les exemptions de péage accordées pour des raisons politiques ou commerciales.

Les péages sont un autre type de document utile à la recherche (fig. 1). Ils ont participé d'une politique économique menée notamment par l'autorité comtale. En recherchant leur acquisition, les pouvoirs comtaux, municipaux ou seigneuriaux contrôlent d'une certaine façon le commerce provençal et augmentent leurs sources de revenus. Des recherches dans les fonds d'archives provençaux et dans la bibliographie ont permis de rassembler 176 tarifs de péage ou taxes maritimes pour plus d'une cinquantaine de communes de Provence et de régions limitrophes ${ }^{2}$. Les plus anciens de ces droits de passage ou d'entrée qui frappent les marchandises en transit datent du XII ou du XIII ${ }^{e}$ siècle. Ils attestent de la nature des produits taxés et de la valeur de cette imposition à un instant précis. Une condition sine qua non à leur exploitation est que le corpus doit être suffisamment important et représentatif géographiquement et chronologiquement. Si pour le premier point l'objectif est plutôt atteint, concernant la périodisation, la plupart des tarifs récoltés sont datés des XIII ${ }^{e}$ et $\mathrm{XIV}^{\mathrm{e}}$ siècles. Les cartes présentes dans cet article (fig. 4 à 7) sont donc avant tout valables pour cette période. Trois principales unités de mesure sont notées pour les matières métalliques (fig. 2) : la bête de somme ou la charrette utilisée préférentiellement en Haute Provence, la saumée, le long de la moyenne Durance et

$\overline{2}$ Thuaudet 2015, fig. 4. 


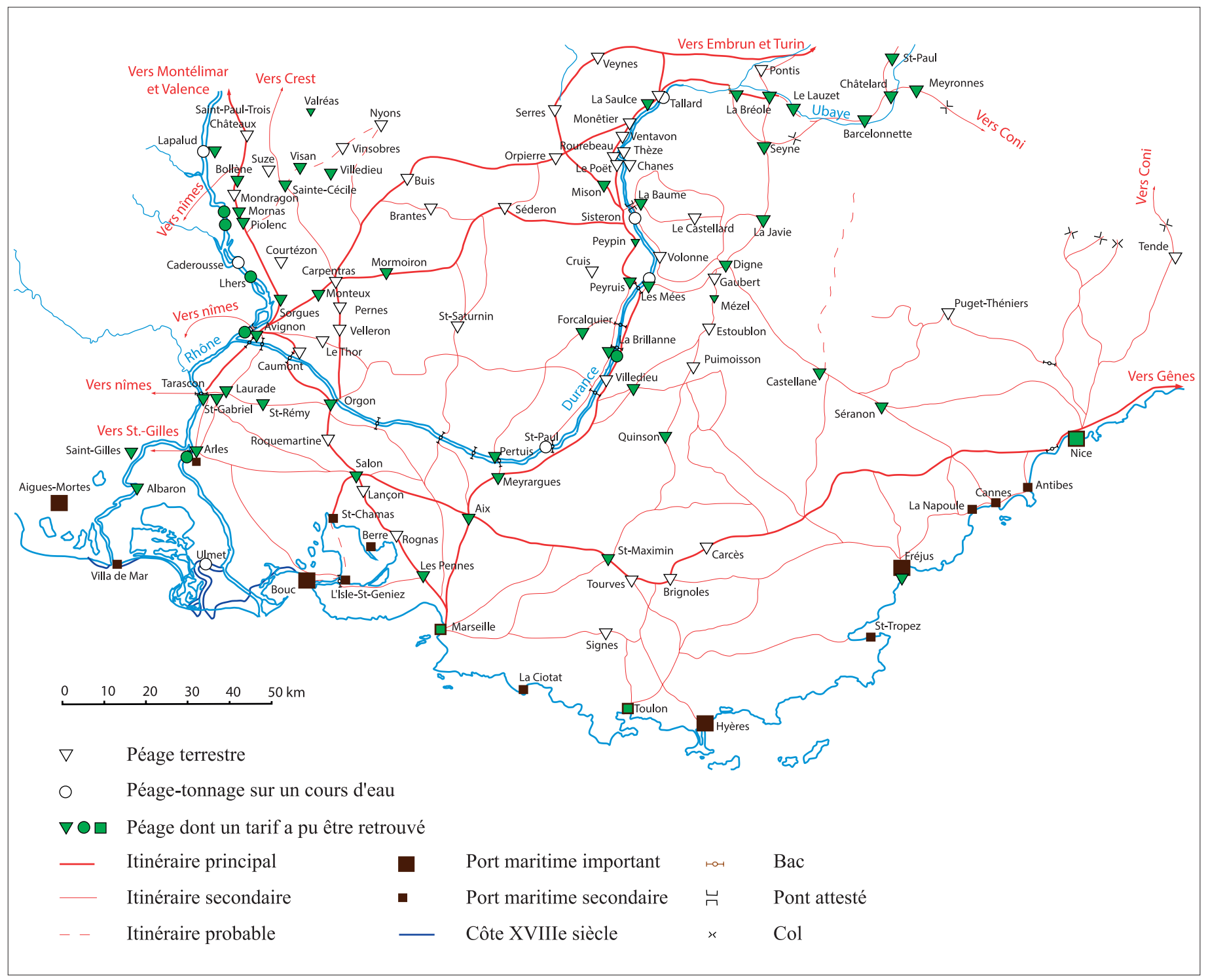

Fig. 1 - Carte des péages provençaux. (d'après Baratier, Ruby, Hildesheimer 1969, carte 86)

à Meyronnes, la charge ou le quintal, en Basse Provence et le long des premiers méandres du Rhône.

La liste des marchandises n'est pas toujours intangible au cours des siècles. Il existe bien des péages pour lesquels les tarifs ne varient guère au cours du temps, où l'imposition reste la même et où la nature des produits n'évolue pas ou peu. D'autres tarifs présentent une certaine part d'immobilisme pendant les XIII ${ }^{e}$ et XIV ${ }^{e}$ siècles, mais changent de manière importante au XV siècle. Les rapports de force entre propriétaires de péage et populations, la coutume ainsi que d'autres facteurs entrent en ligne de compte. Pour de multiples raisons, les tarifs de péage ne correspondent pas forcément à une liste réelle des marchandises qui traversent la zone.

\section{Voies de circulation et péages}

La Provence est parcourue par quatre voies principales, pour certaines autant terrestres que navigables (fig. 1). Une première traverse le territoire d'est en ouest depuis Gênes en passant par Nice, Aix-en-Provence et aboutit à Avignon. Au XIII ${ }^{e}$ siècle, les péages d'Aix exercent un véritable monopole sur les marchandises pour l'ensemble du Comté, qu'elles le traversent d'est en ouest ou du nord au sud. Ils sont complétés par les péages satellites de Valensole et de Saint-Maximin, qui taxent les échanges avec la haute Provence et la Provence occidentale. Il est spécifié que les biens payants à Aix y sont francs et vice-versa. Avec le péage des Pennes, le comte taxe les marchandises sur la route Marseille-Avignon. 


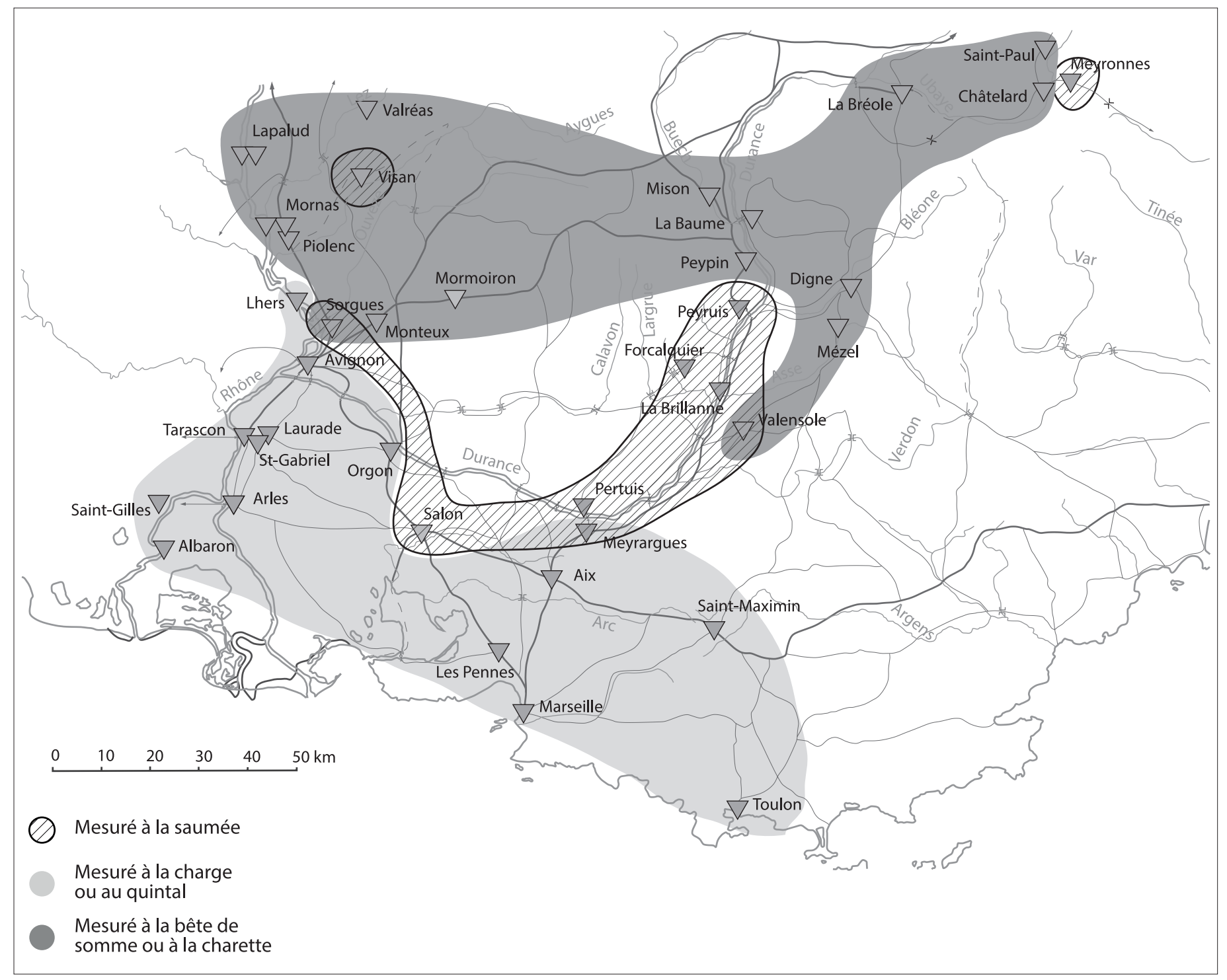

Fig. 2 - Carte des péages provençaux mentionnant du métal.

À hauteur d'Aix-en-Provence, la route est-ouest coupe une route sud-nord qui part de Marseille, court le long de la Durance et permet d'arriver en Savoie, Dauphiné ou Piémont. Des péages le long de la Durance prennent la mesure des échanges entre les différentes parties de la Haute-Provence et contrôlent la descente vers les basses terres. Le commerce avec l'Italie y est encouragé, ainsi ceux qui paient à Châtelard ne paient pas à Meyronnes ou à Larche et inversement.

Une seconde voie sud-nord suit le Rhône. De là, des connexions sont possibles avec Nîmes et le Languedoc. À partir d'Avignon, des routes permettent la liaison avec les Préalpes. Les péages d'Arles et Tarascon, sur le Rhône sont particulièrement rémunérateurs, grâce à leurs tarifs élevés et à leur position stratégique sur cet axe très emprunté par les commerçants de toutes origines. C'est la voie d'accès la plus aisée à partir de la côte provençale vers l'Europe du Nord. Avec Avignon, qui échappe au comte, ces tarifs proposent la plus grande variété de produits. Aux XIII e et XIV ${ }^{e}$ siècles, à Tarascon, un péage se prend aux portes de la ville. Il s'y ajoute un petit péage sur les denrées du commerce local. En outre, vers l'est, les péages de Laurade et de Saint-Gabriel taxent les marchandises qui n'entrent pas dans la cité. À l'ouest, le péage du pont entre Beaucaire et Tarascon, aux taxes peu élevées, porte bien plus sur les passages des animaux, des hommes et des charrettes que sur ceux des denrées. Le péage du Rhône s'intéresse aux marchandises montant ou descendant le fleuve tandis que celui de l'île de Lubières contrôle vraisemblablement un bras secondaire. Pour la même période, il existe à Arles le péage du Bourg-Vieux, celui de La Trouille, 
un quartier au nord de la ville, celui du Levant d'une durée de vie très limitée, le péage du pont de Trinquetaille à l'ouest et le péage du Rhône de même nature que celui de Tarascon. Sur un autre bras du Rhône, le péage de Saint-Gilles à la frontière du Languedoc est, au moins au XII ${ }^{\text {e }}$ ou XIII e siècle, éclaté en trois points de levée dont deux sur des bras secondaires du fleuve ${ }^{3}$.

Bien évidemment, les péages sont majoritairement situés le long des axes principaux, mais un fin réseau secondaire complète le tout. La documentation retrouvée laisse apparaître plusieurs espaces aux renseignements plus rares. Très peu de tarifs ont été récoltés pour la voie entre Aix et Nice ainsi qu'entre le Comtat-Venaissin et le Buech. De même, les relations avec le Roussillon sont assez mal discernées ${ }^{4}$.

\section{Le contexte économique}

Les $\mathrm{XI}^{\mathrm{e}}$ et $\mathrm{XII}^{\mathrm{e}}$ siècles marquent le début d'un essor économique qui atteint son apogée au milieu de la seconde moitié du XIII ${ }^{\mathrm{e}}$ siècle pour se stabiliser ensuite durant un tiers de siècle ${ }^{5}$. Les sources documentaires et archéologiques mettent en évidence une forte croissance de l'activité minière durant cette période. Cette phase d'exploitation intense perceptible sur le pourtour méditerranéen est suivie d'une grande dépression au milieu du XIV ${ }^{\mathrm{e}}$ siècle ${ }^{6}$. Elle est précédée de peu d'une contraction de l'économie médiévale à compter des environs de $1315^{7}$. L'activité minière ne s'interrompt cependant pas totalement, hormis peut-être dans les régions où celle-ci était moins intense et donc plus fragile, comme en Provence. À partir du milieu du XV $\mathrm{XV}^{\mathrm{e}}$ siècle, une politique incitative de prospections est menée avec des résultats variés selon les régions. Comme presque toujours, dans le sud-est de la France, la recherche de l'argent guide la plupart des travaux, mais le plomb ou le cuivre des minerais polymétalliques sont certainement plus rentables, car plus abondants, même en prenant en compte l'écart de prix entre les matériaux.

\subsection{L'étain}

La provenance de l'étain est rarement renseignée dans les sources d'archive. Le sud-est de la France et les régions immédiatement voisines sont presque totalement vierges de

3 Concernant la localisation des péages de Saint-Gilles, voir Bondurand 1901.

$4 \quad$ Une carte répertoriant les péages en Provence est disponible dans Baratier, Ruby, Hildesheimer 1969, carte 86. Se reporter à Thuaudet $2015, t^{\circ} 3$, fig. 4 , pour un inventaire des 176 tarifs de péage consultés pour la rédaction de cet article.

5 Pour la Provence : Février 1964, 213 ; Pernoud 1951, 131-136; Poly 1976, 214-223; Weiberger 1990, 12. Pour l'Europe : Bois 2000, 45-63.

6 Bailly-Maître 2007, 22.

7 Bois 2000, 62-64. gisement d'étain ${ }^{8}$. Ce matériau fait donc l'objet d'un trafic à longue distance (fig. 3). L'étain est un produit d'importation. Il provient essentiellement d'Angleterre et notamment de la région des Cornouailles dont les gisements sont les plus purs et les plus abondants en Europe ${ }^{9}$. Dans la seconde partie du Moyen Âge, l'expédition du métal vers le continent se fait principalement vers le sud-ouest de la France par les ports de La Rochelle, de Bordeaux, de Bayonne et Oléron ${ }^{10}$. Toutefois, une part conséquente des exportations se fait également vers la Normandie et les Flandres. Plusieurs voies de transport de l'étain sont possibles pour l'acheminer en Provence. L'une d'elles passe par Bordeaux, remonte la Garonne jusqu'à Toulouse, d'où la marchandise est transbordée sur des animaux de bât qui la conduisent jusqu'à Narbonne. Ce cheminement est décrit par le géographe arabe Ibn Saîd au XIII ${ }^{e}$ siècle ${ }^{11}$. De ce port, l'étain peut être redistribué en Méditerranée, vers Marseille ou Arles par exemple. Un cheminement terrestre a pu exister depuis Toulouse, en passant par Montpellier, pour faire parvenir l'étain à Arles, Avignon ou Tarascon. L'absence de la mention de l'étain avant le $\mathrm{XV}^{\mathrm{e}}$ siècle dans le tarif du pont de Trinquetaille à Arles contrarie quelque peu cette hypothèse. Une seconde voie, bien plus difficile à matérialiser, débute dans le nord de la France et aboutit dans la vallée du Rhône.

À la fin du XIII siècle, le quasi-monopole anglais dans la distribution de l'étain est troublé par les gisements de Bohême et de Saxe qui augmentent leur production de manière conséquente ${ }^{12}$. Les marchands du sud-ouest de la France perdent de leur importance dans la diffusion de l'étain du fait de cette concurrence en provenance d'Europe Centrale. De leur côté, les Allemands sont concurrencés par les négociants italiens qui s'approvisionnent désormais directement dans les Flandres et monopolisent peu à peu le trafic de l'étain anglais, qui reste malgré tout soutenu ${ }^{13}$. Cela se traduit par des transports d'étain depuis l'Italie vers la Provence ${ }^{14}$.

L'étain d'Europe centrale arrive en Provence par la vallée du Rhône, par l'intermédiaire de commerçants allemands. Les données récoltées montrent toutefois que

8 Des travaux récents ont mis en évidence une exploitation du minerai d'étain en alluvion dans le district de la Viadène en Aveyron qui se termine au haut Moyen Âge. Renseignement communiqué par Philippe Abraham.

9 Hatcher 1973, 3 ; Malham 2010.

10 Hatcher 1973, 22.

11 Hatcher 1973, 24.

12 Hatcher 1973, 25-26, 103.

13 Hatcher 1973, 91, 95-102.

14 À la fin du XIV ${ }^{e}$ siècle, la compagnie Datini procède à des envois depuis Pise jusqu'à Arles puis Avignon en remontant le Rhône (Antonietti 2007, 172-175, 789-798). En 1485, un bâtiment génois chargé entre autres d'étain à destination d'Arles est saisi par les Marseillais (Baratier, Reynaud 1951, 198, n.2). 


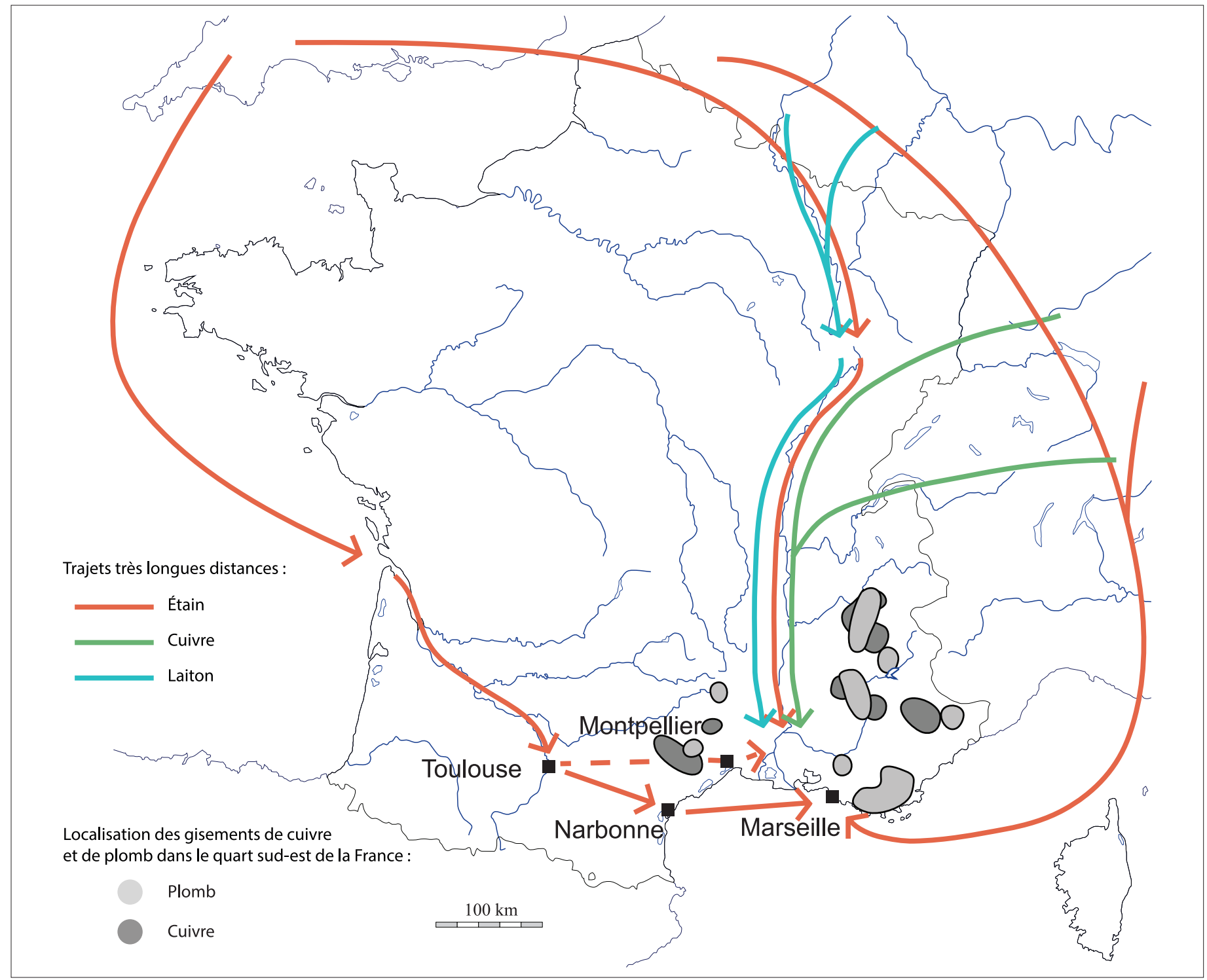

Fig. 3 - Circulation des métaux.

cette source d'approvisionnement est bien plus tardive que l'étain anglais ${ }^{15}$. Elle n'intervient qu'après le doublement de la capacité de production de ces gisements au début du $\mathrm{XVI}^{\mathrm{e}}$ siècle ${ }^{16}$. À la fin du XVI ${ }^{e}$ siècle et au début du XVII siècle, une part de l'étain qui arrive à Marseille est encore originaire d'Angleterre, transportée par des Anglais ou des Bretons par voie maritime ${ }^{17}$.

En Provence, de nombreux tarifs de péages attestent de l'importance des échanges sur ou le long du Rhône (fig. 4). Sans surprise, les tarifs de péage de la moyenne Durance

15 Collier, Billioud 1951, 211, 518-521.

16 Hatcher 1973, p. 121.

17 Collier, Billioud 1951, 245, 459, 518-521; Bergasse 1954, 173. comportent peu d'attestations de l'étain. Elles sont sans doute liées à une activité de redistribution de la matière première dans l'arrière-pays, depuis l'axe Marseille-Pertuis ou par des voies transversales comme celle passant par Mormoiron, ou en remontant la Durance. Dans le Var, Toulon a pu servir de point d'approvisionnement. Le commerce de l'étain en Provence est peu renseigné dans les archives notariales et ne dépasse pas quelques quintaux ${ }^{18}$ malgré de fortes concentrations de potiers d'étain notées lors de dépouillements aux archives pour Aix-en-Provence, Avignon et Draguignan et sa région. Les potiers d'étain sont les principaux acteurs de son commerce

18 Voir Schäfer 1937, p. 197 ; AD BDR Aix, 309 E 116, fo $260 \mathrm{r}^{\circ}-260 \mathrm{v}^{\circ}$, 30 juillet 1421 ; AD BDR Aix, 307 E 12, n.f., 24 janvier 1423 ; 307 E 82, fo $176 v^{\circ}, 20$ avril 1447. 


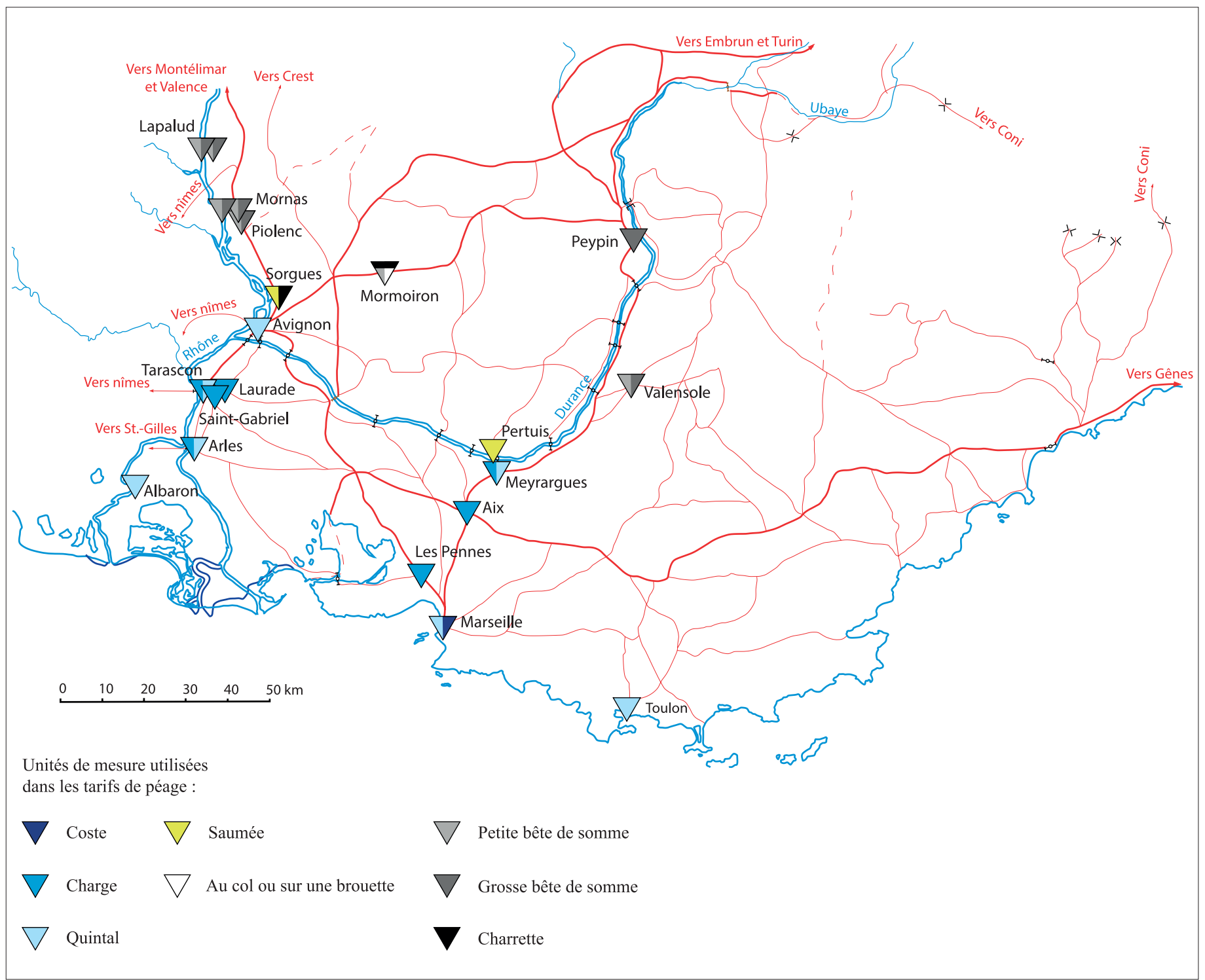

Fig. 4 - Carte des péages provençaux mentionnant l'étain.

en Provence. La récupération de vieil étain à Marseille et même ailleurs en Provence par les artisans marseillais est renseignée par quelques sources d'archives ${ }^{19}$. Elle dut être beaucoup plus importante que ne le suggèrent celles-ci et commune à l'ensemble des artisans provençaux, car l'étain ainsi récolté est d'un prix moins élevé que l'étain neuf ${ }^{20}$. À la fin du XIVe siècle et dans la première moitié du siècle suivant, le négoce d'exportation se fait principalement vers le Levant et notamment Beyrouth ${ }^{21}$, mais les données sont rares. Dans la seconde moitié du XVI e siècle, les exportations

\footnotetext{
19 Baratier, Reynaud 1951, 662-663, 715, 827.

20 En 1430, à Dijon, le fin estaing est deux fois et demi plus cher que le mort estaing (Chapelot 1985, p. 309).

21 Baratier, Reynaud 1951, 72, 237, 246, notes 3 et 4, 715.
}

d'étain s'intensifient en raison d'évènements militaires : les guerres entre Venise et les Turcs, alliés du royaume de France. Quelques milliers de quintaux quittent ainsi le port de Marseille ${ }^{22}$, alors certainement l'un des pôles les plus importants du commerce de ce matériau en Méditerranée, grâce à sa position géographique. La cité phocéenne est en outre le lieu de nombreuses fontes d'artillerie - qui nécessitent des alliages cuivreux - dans la seconde moitié du XVI ${ }^{e}$ siècle ${ }^{23}$.

Létain (stagnum, latin, estan, français ou provençal, staing, provençal) peut être transporté sous forme de plaques

22 Collier, Billioud 1951, 211, 247, 260, 518-521.
23 Collier, Billioud 1951, 521-524. 
(lama, toscan $)^{24}$, de verges (vergha, toscan, virga, latin, verga, provençal) ${ }^{25}$, de lingots de fonte (stagnum gitatum, latin) ${ }^{26}$, en saumon (samon, provençal) ${ }^{27}$, en « cloche » $(\text { cloca })^{28}$, en pièces (pessa, provençal) ${ }^{29}$ et conditionné en « ballons " pesant $1,5^{30}$ ou 1,7 quintal ${ }^{31}$ ou en caisses de 2,3 quintaux ${ }^{32}$. La forme de la matière première influe sur la valeur marchande de l'étain. Une unique dénomination de qualité a pu être repérée : l'étain fin (finum) ${ }^{33}$.

\subsection{Le plomb}

L'extraction du plomb a été pour la plupart réalisée dans le cadre de l'exploitation de minerais polymétalliques à teneur en argent (fig. 3). La distribution des mines est donc pour partie identique à celle du métal précieux. Entre le $\mathrm{XI}^{\mathrm{e}}$ et le $\mathrm{XIV}^{\mathrm{e}}$ siècle, la Savoie et le Dauphiné sont les principales zones d'activité ${ }^{34}$, mais les régions plus méridionales du massif ont aussi été l'objet de travaux plus spécifiques, de faible ampleur, parfois sur des gîtes de plomb seul ${ }^{35}$. Les mines de Brandes, en Isère, exploitées entre le XII et le début du $\mathrm{XIV}^{\mathrm{e}}$ siècle constituent l'un des principaux centres médiévaux de production de plomb argentifère ${ }^{36}$. Après la déprise du XIV ${ }^{e}$ siècle suit une période de faible activité ainsi que l'indique par exemple le faible bénéfice tiré de l'albergement des mines de plomb et de plomb argentifère de l'Oisans ${ }^{37}$. Des sources d'archives attestent à la fin du XV $\mathrm{XV}^{\mathrm{e}}$ siècle et au début du XVI ${ }^{e}$ siècle d'un renouveau de la recherche du minerai de plomb argentifère dans les Alpes-de-Haute-Provence ${ }^{38}$. Cette activité s'accroît à partir de la fin du XVI siècle et perdure jusqu'au début de l'Époque moderne, accompagnée

\section{Antonietti 2007, 172-175, 789-798.}

25 Blancard 1884-1885, to 1, doc. 209 ; Baratier, Reynaud 1951, 243 ; Antonietti 2007, 172-175.

26 Blancard $1884-1885, \mathrm{t}^{\circ} 1$, doc. 194 et 298.

27 Ce terme provençal est repris dans un texte en latin : stagni dicti de samon (AD BDR Aix, $308 \mathrm{E} 712$, fo $15 \mathrm{r}^{\circ}, 24$ janvier 1504).

28 Blancard 1884-1885, $\mathrm{t}^{\circ} 1$, doc. 209 et 779.

29 AD Vaucluse, CC 1008, fin XIV ${ }^{\mathrm{e}}$ siècle.

30 Baratier, Reynaud 1951, 243.

31 Collier, Billioud 1951, 520.

32 Collier, Billioud 1951, 519

33 AD BDR Aix, 307 E 12, n.f., 24 janvier 1423 ; 307 E 82, fo 176 Vº 20 avril 1447.

34 Sclafert 1926, 539 ; Ancel 1998; Bailly-Maître, Dhénin 2004, 45, 48 ; Ancel 2006a ; Ancel 2006b, 163-164 ; Ancel 2010, 294-295.

35 Becquey 1829, 210 ; Ancel 2006a. De nombreux gîtes répertoriés par Roux 1862, 203-204, 207, 208, 213, 218, ne présentent pas de traces d'exploitation anciennes. Bailly-Maître, Dupraz 1994 ; Bailly-Maître, Dhénin 2004 ; Bailly-Maître 2007.

37 Sclafert 1926, 541-542.

38 Becquey 1829, 211 ; Féraud 1861, 130 ; Collier et Billioud 1958, 42-43 ; Morin et Guiomar 2006; Ancel 2010, 296. de quelques tentatives d'exploitation. Souvent le plomb extrait n'est jugé bon qu'à servir d'alquifoux aux potiers ${ }^{39}$. Aux XVI ${ }^{e}$ et XVII siècles, la région du Buëch dans les HautesAlpes et le massif des Hurtières en Savoie font également l'objet de prospections et d'exploitations ${ }^{40}$. En Piémont, des études récentes ont mis en évidence l'existence de quelques gisements exploités au bas Moyen Âge et au début de l'Époque moderne ${ }^{41}$.

En Provence, l'essentiel des activités minières avérées, médiévales et du début de l'Époque moderne, est concentré dans le massif des Maures dans le Var, et dans les environs de Toulon ${ }^{42}$. En 1180, un contrat signé entre des «argentarii » exploitant une mine de plomb argentifère au terroir de Toulon, le comte de Provence, Raymond Bérenger IV, et le vicomte de Marseille, attribue à chacune des parties un tiers des revenus de la mine ${ }^{43}$. Le plus important gisement de plomb argentifère est assurément celui des Bormettes dans le massif des Maures, site pour lequel des textes des XIII et $\mathrm{XIV}^{\mathrm{e}}$ siècles attestent d'une activité minière ${ }^{44}$. En 1433 , deux associés, un marchand avignonnais et un épicier du diocèse de Fréjus, possèdent des mines de plomb argentifère, l'une à Cogolin, l'autre à La Garde-Freinet ${ }^{45}$. À partir du second tiers du XV siècle, les prospections et autorisations d'exploitations se multiplient. Cependant, les documents explicitent rarement quels sont les métaux dont l'extraction est prévue et ne donnent pas toujours une localisation très précise ${ }^{46}$. La confrontation des archives avec les données de terrain est utile. Elle indique que les lettres royales obtenues par Antoine Payant en 1478 pour la recherche et la mise en valeur des minéraux du terroir du Luc

39 Le récit de la visite de $\mathrm{M}$. Darluc dans les Alpes et Préalpes est fort instructif sur les tentatives modernes d'extraction et sur la qualité du minerai (1784, p. 109-119, 254-255, 274, 296-297, 314-315, 345)

40 Ancel 2010, 296 ; Crabières 2001, 60-65.

41 Di Gangi 2001, fig. 2 ; Di Gangi 2007, fig. 3.

42 Présence d'une mine de plomb argentifère dans le terroir de Toulon en 1180 (B 289, parchemin). Document mentionné dans Blancard 1868 (p. 33-34), Benoit 1960 (p. 228) et Berthet 2007 (p. 65). En 1433, deux associés, un marchand avignonnais et un épicier fréjussien, possèdent des mines de plomb argentifère, l'une à Cogolin et l'autre à La GardeFreinet (AD Var, 3 E 8 787, f॰ 57 et 58).

43 B 289, parchemin. Document mentionné dans Blancard 1868, p. 33-34 ; Benoit 1960, p. 228 ; Berthet 2007, p. 65.

44 Ancel 2010, 295.

45 AD Var, 3 E 8787 , f० 57 et 58.

46 Par exemple, une convention signée à Aix-en-Provence, en 1470, mentionne cinq techniciens « allemands » originaires des diocèses de Nuremberg, Salsbourg, Freising, Prague et pour le dernier d'origine inconnue, recrutés par deux associés pour extraire par fusion, au moyen d'un four de traitement à soufflerie hydraulique (fozina... an lo molin), l'or du cuivre et l'argent du cuivre et du plomb (Coulet 1975, 160161, 167 ; Verna 2017, 388). En avril 1504, cinq mineurs à la recherche de minerais et principalement semble-t-il de plomb et de cuivre, s'engagent à présenter tous les six mois à la Cour un compte destiné à servir de base aux prélèvements royaux (AD BDR Aix, B 1234, f० $7 \mathrm{r}^{\circ}$ $\left.-7 v^{\circ}\right)$. 
ont pu concerner des gîtes de fer ou de plomb argentifère ${ }^{47}$. En 1502, une société regroupant un marchand et trois orfèvres provençaux se constitue avec pour objectif principal l'extraction d'or et d'argent, au quartier de Saint-Daumas, au Cannet ${ }^{48}$. Or cette zone est particulièrement connue pour ses gisements de plomb argentifère. Selon M.P. LanzaBerthet, des travaux miniers au pic et au feu sur le filon $\mathrm{T}$ et dans la concession de Pic-Martin, sur la commune du Cannet pourraient correspondre ${ }^{49}$. Début janvier 1580, un marchand du Piémont et un peintre d'Aix s'associent pour quatre ans pour l'exploitation de deux gisements sur des terrains qu'ils ont pris en location ${ }^{50}$. Le premier est une myniere du vernys et plomb à la montagne de Bayon apelle Roynon, sur le terroir de Bayon, qu'ils tiennent à part égales. Le second, dont le peintre Lomellin ne possède qu'un tiers des parts, est au village de Dremont, sur la montagne Arpil. Il est ici clairement fait le choix de mettre en œuvre des travaux destinés à obtenir une qualité spécifique de plomb adaptée à la peinture et à d'autres usages non métallurgiques. Des fouilles réalisées à Évenos, ont révélé une installation de traitement du plomb, malheureusement non datée ${ }^{51}$. Dans la première moitié du XVII siècle, l'attention portée au plomb se concrétise par des essais de minerai et l'extraction d'un matériau destiné avant tout à servir à la glaçure des céramiques ${ }^{52}$.

L'exploitation du plomb dans le midi de la France est peu connue malgré la présence de gîtes de plomb et de plomb argentifère dans la Lozère, le Gard, l'Hérault, l'Aude et les Pyrénées-Orientales ${ }^{53}$. La recherche de minerai de plomb argentifère est attestée au Moyen Âge dans la région de Lodève ${ }^{54}$ et son exploitation connue autour du Mont Lozère entre le XI et le début du XIV ${ }^{e}$ siècle ${ }^{55}$. La production locale ne devait pas être très importante si l'on en juge par le transfert en 1367 de presque 200 quintaux qui partent d'Avignon par bateau pour Montpellier en passant par Lattes, pour la construction d'un monastère ${ }^{56}$. Les quelque 114 quintaux de plomb acquis au milieu du XIV ${ }^{e}$ siècle à Montpellier et destinés aux bullateurs papaux avignonnais, dont une partie arrive à Comps par voie terrestre avant d'être transbordée sur

47 Omnia mineralia sive mineras ferri, calibis, eris, plumbi, stagni, esmerighi, etc. (Coulet 1975, 165). Coulet 1975, 165)

49 Lanza-Berthet 2006, 153.

50 AD BDR Aix, 303 E 132, fo $44 \mathrm{v}^{\circ}-47$ r $\mathrm{r}^{\circ}$ (Thuaudet 2015, annexe 8, doc. 2).

51 Serra 1992. Un prélèvement pour une datation au C14 aurait été réalisé, mais les résultats ne sont pas connus.

52 Thuaudet 2015, 120-121.

53 Becquey 1829, 212-214 ; Lechelon 1997, 167.

54 Lopez 1989.

55 Bailly-Maître 2007, 28 ; Bailly-Maître 2010, 139.

56 Schäfer 1937, 195. une barque remontant le Rhône ${ }^{57}$, pourraient être d'origine plus lointaine : de l'arrière-pays languedocien, des Pyrénées ou bien de la Péninsule ibérique. En effet, en mai 1508, un capitaine de bateau marseillais charge à Alicante dix quintaux de plomb ${ }^{58}$.

Aux ressources, si ce n'est locales, du moins géographiquement proches, semblent s'être ajoutées ponctuellement des importations de plomb de régions plus lointaines. C'est le cas du plomb de Sardaigne qui, au $\mathrm{XIV}^{\mathrm{e}}$ siècle, arrive à Marseille ${ }^{59}$ ou remonte le Rhône jusqu'à Avignon ${ }^{60}$ pour servir aux besoins de la cour pontificale ${ }^{61}$. Du plomb anglais est débarqué par des vaisseaux bretons et anglais en 1589 et $1590^{62}$. Dans la première moitié du $\mathrm{XVII}^{\mathrm{e}}$ siècle, l'Angleterre est le principal fournisseur de Marseille en plomb, mais il en vient également de la Péninsule ibérique, d'Allemagne et des Pays-Bas, et d'Arles par la voie du Rhône ${ }^{63}$. Peut-être parvient-il en Provence du plomb anglais avant cette date même si les sources provençales consultées sont pour le moment muettes. Marseille sert bien évidemment de pôle de redistribution, vers Saint-Chamas et Ollières en $1426^{64}$, mais aussi Arles, Beaucaire, Toulon, mais plus loin également vers Narbonne et même Barcelone dans la première moitié du XVII ${ }^{e}$ siècle ${ }^{65}$.

Pour la période d'étude, l'ampleur de la circulation du plomb à la descente du Rhône ou traversant le Rhône n'a pu être établie, au moyen d'un sondage dans des comptes de péages, que pour les péages terrestres et fluviaux d'Arles, pour le dernier tiers du XVI siècle et le premier tiers du siècle suivant ${ }^{66}$, époque où Arles est en plein renouveau économique ${ }^{67}$. Le plomb est le seul métal qui apparaît et sa circulation, quoiqu'irrégulière, est tout de même assez fréquente. Si on en juge par les tarifs de péage, les vallées du Rhône et de la Durance jouent leur rôle habituel dans le commerce du métal (fig. 5). Le tarif de Saint-Maximin du milieu du XIV e siècle conserve peut-être la trace de la circulation du plomb issu des travaux miniers varois. Quoi qu'il en soit, alors que la taxation des autres métaux est ordinairement la même en Provence, à l'exception de l'or et de l'argent bien plus imposés, un tarif de faveur est souvent

\footnotetext{
57 Schäfer 1914, 315, 504 et 540 .

58 Collier, Billioud 1951, 115.

59 Baratier, Reynaud 1951, 147.

60 Schäfer 1937, 15, 48.

61 Schäfer 1937, 55, 136, 195.

62 Colllier, Billioud 1951, 245, 459, note 7, 518.

63 Bergasse 1954, 172.

64 Collier Billioud 1951, 662.

65 Bergasse 1954, 172 ; Collier, Billioud 1951, 662.

66 AD BDR Marseille, 3 G 60, fo 683 à 1323.

67 Payn-Echalier 2006, 195.
} 


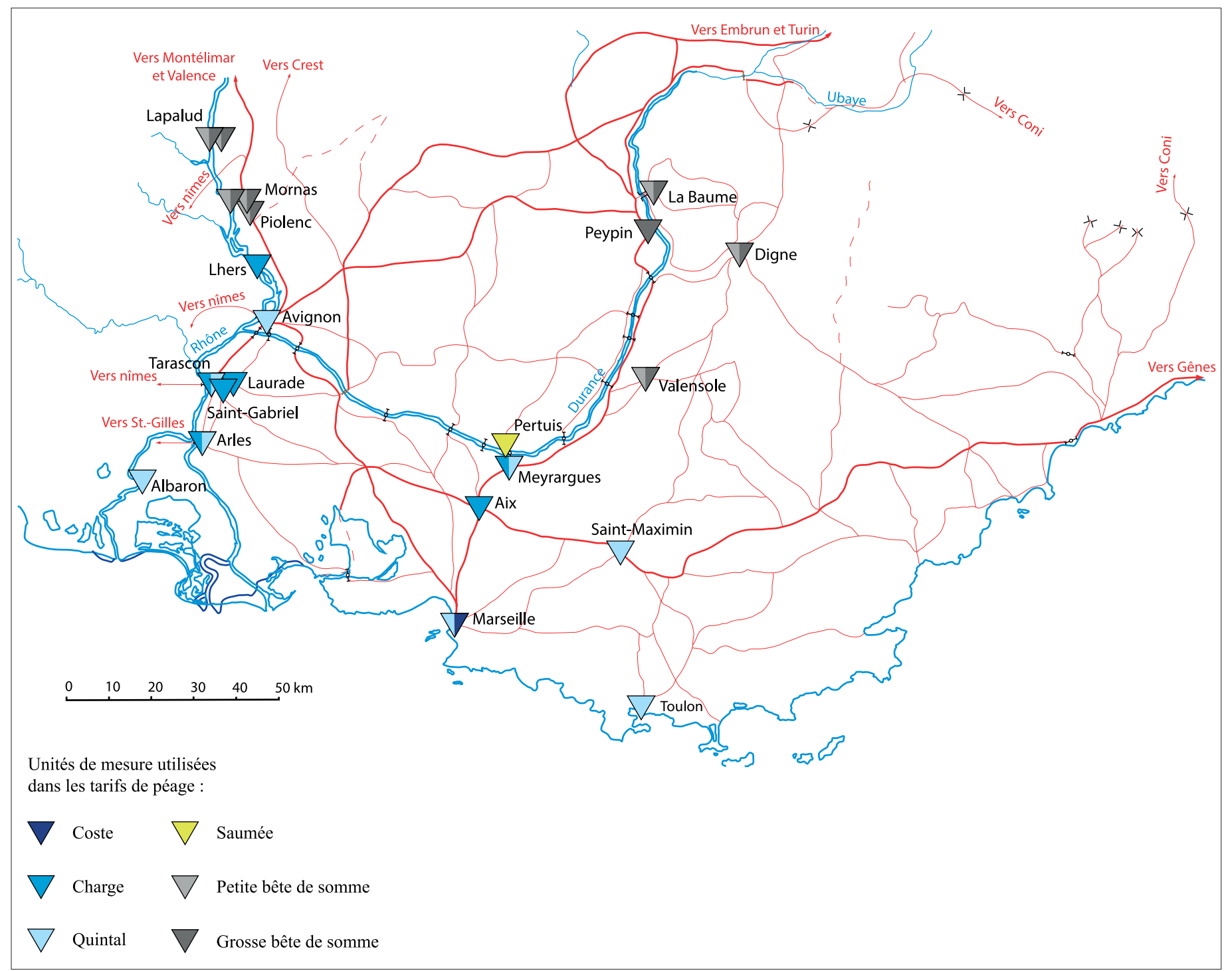

Fig. 5 - Carte des péages provençaux mentionnant le plomb.

octroyé au plomb. Il est ainsi parfois taxé de deux à trois fois moins ${ }^{68}$. Est-ce parce qu'il s'agit d'un métal produit essentiellement localement? Cela est fort possible. Ce métal est également très courant et donc peu cher, car produit en très grande quantité notamment par l'exploitation intensive des gites de minerais de plomb argentifère. Il n'a pas non plus une importance stratégique. Il est curieux de constater que rares sont les mentions de la forme de la matière brute : il est vendu sous forme de pecias sive lamina ${ }^{69}$, de lingots (molles, provençal) ${ }^{70}$, de pains ${ }^{71}$. Aucune indication de forme n'est donnée dans les tarifs de péage. Le recyclage du plomb

\footnotetext{
68 Thuaudet 2015, fig. 10, 12, 14, 16.

69 Schäfer 1937, 195, 540.

70 Bernardi 1990, 252.

71 Collier, Billioud 1951, 149.
}

n'a pas laissé de trace dans les archives dépouillées, mais il doit cependant tenir une place importante dans la matière première utilisée par les artisans provençaux, d'autant plus que le point de fusion de ce métal est bas.

\subsection{Le cuivre et ses alliages}

Le cuivre est, dans le sud-est de la France, ordinairement extrait de sulfates et de carbonates et souvent associé au plomb ou à l'argent (fig. 6). Si des indices de cuivre se rencontrent couramment dans les Alpes ${ }^{72}$, le massif des Maures, les Cévennes, la Montagne Noire, les Corbières et la partie

72 Bonnaire 1802, 102-103, Becquey 1829, 210 ; Darluc 1784, 63-64, 113, 262, 264, 274, 296-297. 


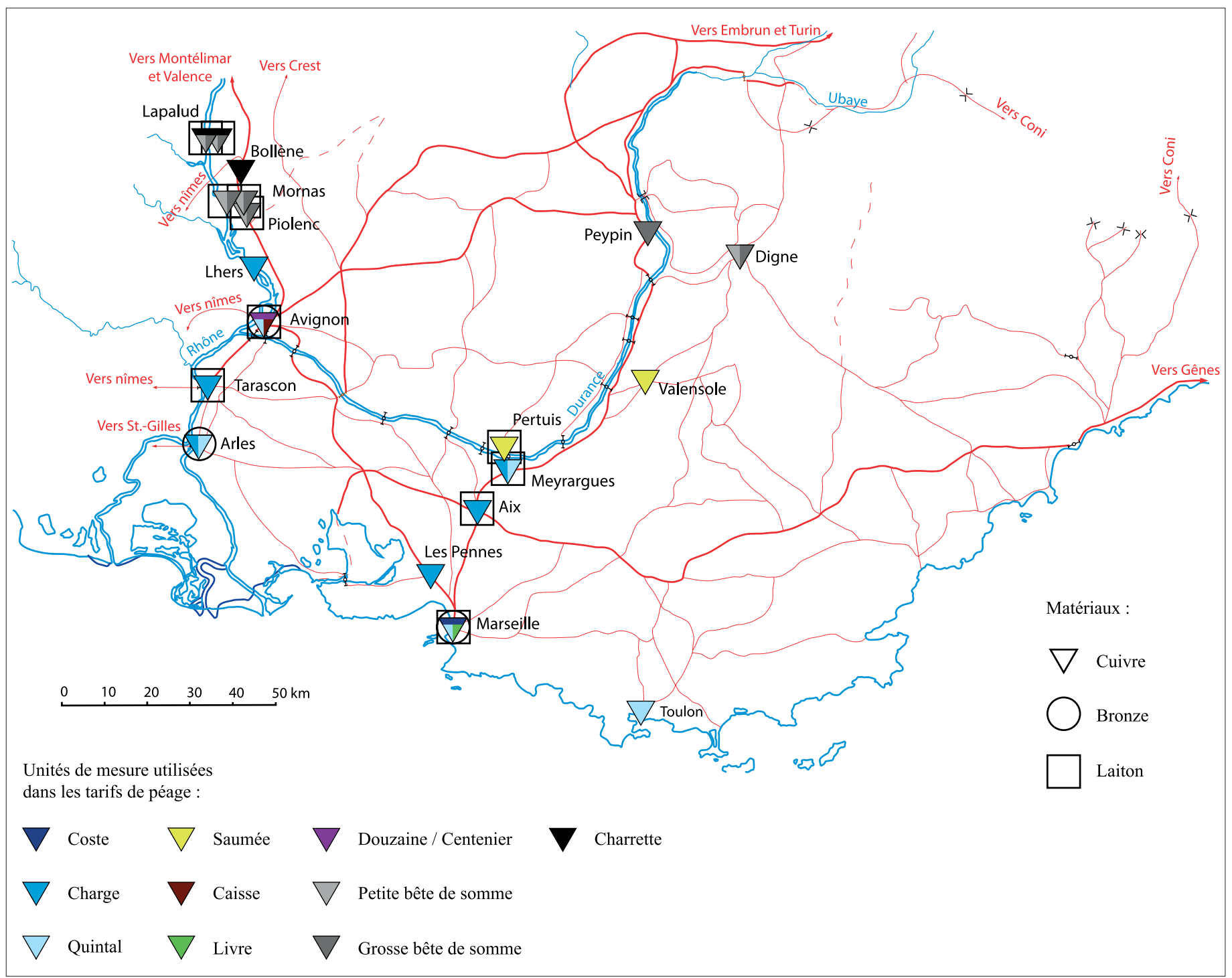

Fig. 6 - Carte des péages provençaux mentionnant le cuivre et ses alliages.

orientale des Pyrénées ${ }^{73}$, ceux-ci n'ont souvent pas pu donner lieu à une exploitation dans la seconde partie du Moyen Âge et à l'Époque moderne. La plupart des gisements médiévaux exploités sont localisés dans les Alpes et notamment sa partie nord, l'Oisans et la Savoie ${ }^{74}$. L'exploitation des gisements de cuivre argentifère d'Aiguebelle et d'Hurtières permet l'extraction de plus d'une dizaine de milliers de quintaux ${ }^{75}$. Mais l'activité diminue très sensiblement par la suite: quelques redevances sont encore perçues sur le cuivre par les comtes de Savoie au XV $\mathrm{XV}^{\mathrm{e}}$ siècle ${ }^{76}$. Il est probable que cette activité extractive du cuivre ait continué à une faible échelle, au XVI ${ }^{\mathrm{e}}$ siècle, grâce au développement du saigerprozess ${ }^{77}$. Dans la partie sud des Alpes, des travaux sont attestés aux $\mathrm{XV}^{\mathrm{e}}$ et $\mathrm{XVI}^{\mathrm{e}}$ siècles $^{78}$. En Piémont, quelques mines en activité sont connues pour la fin du Moyen Âge et le XVI siècle $^{79}$.

De nombreux gites de cuivre sont localisés dans le Massif varois, mais il n'a pas été mis en évidence pour le moment de preuve d'une exploitation médiévale ou du tout début
73 Barge 2006, 12, fig. 1.

74 Sclafert 1926a, 539 ; Bailly-Maître 2000; Crabières 2001, 66 ; BaillyMaître et Dhénin 2004, 48 ; Ancel 2010, 295.

75 Garioud 2007, 51 ; Benoit, Braunstein 1983, 186, 188.

76 Garioud 2007, 55, note 37.
77 Voir au sujet du saigerprozess, Guillot et Benoit 1994, 118

78 Billioud 1958, 43; Barge 2006, 18 ; Ancel 2010, 296. Visite aux mines de plomb de Barles en 1602 (AD BDR Aix, B 1315, $\mathrm{f}^{\circ} 1527 \mathrm{r}^{\circ}-1533 \mathrm{r}^{\circ}$ ).

79 Di Gangi 2001, fig. 2 ; Di Gangi 2007, 79, fig. 3. 
de l'Époque moderne ${ }^{80}$, et ce malgré les nombreuses autorisations de prospections accordées durant cette période par l'autorité comtale puis royale. Pourtant, l'abondance des travaux miniers réalisés à partir du XVIII siècle sur les gisements de cuivre et de cuivre argentifère ${ }^{81}$ laisse penser que des tentatives d'exploitation ont très certainement été menées dans des périodes plus anciennes ${ }^{82}$.

Plus à l'ouest, dans le Gard, notamment dans le district d'Hierle ${ }^{83}$, dans l'Hérault et dans l'Aveyron, des mines de cuivre argentifère ont été exploitées ou l'ont probablement été durant la période d'étude ${ }^{84}$. Du cuivre acheté à Toulouse parvient à Marseille en $1248^{85}$. Peut-être vient-il de l'arrièrepays ou de la Péninsule ibérique.

Les exploitations minières évoquées ci-dessus n'ont pas une production suffisante pour couvrir la consommation de cuivre provençale et des régions voisines des centres d'extraction. Des importations massives sont donc nécessaires, mais les informations obtenues dans la bibliographie et les archives sont assez réduites (fig. 3). Quelques rares transports de cuivre depuis Pise ${ }^{86}$ - peut-être extrait de Toscane ${ }^{87}$ - et la Sardaigne ${ }^{88}$ sont attestés aux XIV et XV ${ }^{e}$ siècles. En 1575, du cuivre de rosette que l'Ordre de Malte veut importer depuis Marseille est dit d'Allemagne ${ }^{89}$. Au début du siècle suivant, le cuivre, le laiton et le bronze arrivent depuis Hambourg ou du Lyonnais et du Beaujolais par la vallée du Rhône ${ }^{90}$.

Il est fort probable qu'une partie conséquente du cuivre travaillé en Provence soit originaire d'Europe centrale. Des mines hongroises, le grand capitaliste allemand Jacob Fugger et ses associés font extraire annuellement, fin $\mathrm{XV}^{\mathrm{e}}$ - début $\mathrm{XVI}^{\mathrm{e}}$ siècle, plusieurs dizaines de milliers de quintaux. La majeure partie approvisionne les marchés d'Europe de l'Est, mais de grandes quantités sont aussi expédiées au marché international de Venise où il part pour l'Orient ${ }^{91}$. Plusieurs

80 G. Mari affirme sans preuve qu'une exploitation de cuivre a été menée au XIV e siècle sur le site du Peirol (Mari 1979, 127). Les traces de pic qui y ont été retrouvées ne sont pourtant pas datées comme le rappelle M.-P. Lanza (1997, 197).

81 Papon 1780, 230, 288 ; Becquey 1829, 210-211; Noyon 1846, 59 , 65 ; Darluc 1876, 296 ; Lanza 1997, 57-59, 83, 88, 125 ; Barge 2006, 19 ; Agay 1980.

82 Lanza 1997, 125 et 197.

83 Bailly-Maître 1989, 62 ; Bailly-Maître 2002, 99, 112 ; Bailly-Maître 2007, 26-27; Bailly-Maître 2012.

84 Becquey 1829, 213-216; Léchelon 1997 ; Maas 2002, 59-60.

85 Blancard 1884-1885, $\mathrm{t}^{\circ} 1, \mathrm{n}^{\circ} 780$.

86 Baratier, Reynaud 1951, 186.

87 De nombreux travaux miniers concernant le cuivre y ont été menés. Voir par exemple Casini 2007.

88 Casini 2007, 442.

89 Collier, Billioud 1951, 517.

90 Bergasse 1954, 172.

91 Schick 1957, 280-281. dizaines de milliers de quintaux de cuivre sont aussi extraits du Tyrol ${ }^{92}$. Une grande part de ce cuivre part pour les marchés de Nuremberg et de Venise. Des transports sont également réalisés en direction de Gênes et Milan, et même de Lyon ${ }^{93}$. Alimentée par des marchands allemands, la cité lyonnaise est un centre d'approvisionnement important en cuivre pour le royaume de France ${ }^{94}$ et vraisemblablement pour le Comté de Provence. Des artisans provençaux y viennent en chercher au XVI ${ }^{e}$ siècle ${ }^{95}$. Le cuivre en provenance de Lyon a très certainement approvisionné les martinets à cuivre, nombreux dans le Vaucluse, où il arrive sous forme de disques de matière très épurée issus du fourneau d'affinage (rosetta), de plaques obtenues par la fonte (plata). Il y est transformé en demi-produits susceptibles d'être utilisés par les artisans provençaux ${ }^{96}$. Les tarifs de péage avignonnais identifient le cuivre sous forme de grenaille ou de lingot en forme de pain ou de plaque. Cependant, les cités de Milan et de Florence, approvisionnées à la fois localement et par du cuivre d'Europe centrale, fournissent à la Provence de nombreux demiproduits tels que fils et feuilles métalliques, mais aussi des produits finis ${ }^{97}$.

Pour en revenir au cuivre hongrois, celui-ci a également fait l'objet d'envois massifs vers l'Italie du Nord, mais la documentation marseillaise ne témoigne pas d'un commerce important du métal par voie maritime depuis l'Italie. De même, il semble avoir peu circulé par les cols alpins d'après les tarifs de péage (fig. 6).

Au milieu du XIII ${ }^{e}$ siècle, les quelques exportations de cuivre à partir de Marseille, rencontrées dans le registre de $1248 \mathrm{du}$ notaire Almaric, se font à destination de Naples ${ }^{98}$, d'Acre ${ }^{99}$, ou de Bougie ${ }^{100}$. En 1406, du cuivre est encore envoyé vers le Maghreb ${ }^{101}$. Dans le troisième quart du XV ${ }^{e}$ siècle, du cuivre brut est exporté depuis le port de Bouc ${ }^{102}$. Dans la seconde moitié du XVI ${ }^{e}$ siècle, les mesures protectionnistes édictées sur le plan national par le pouvoir royal, et proscrivant l'exportation des métaux, sont contournées par l'octroi de licences portant sur plusieurs dizaines, centaines ou milliers de quintaux. Dans les faits, les chargements réels furent

92 Schick 1957, 275, 278-281; Braunstein 2016, 353-359, 525-549.

93 Schick 1957, 278-280.

94 Schick 1957, 278.

95 Collier, Billioud 1951, 522. Vente de métal par un fondeur de Lyon à un campanier aixois (AD BDR Aix, 306 E 471, fo $139 \mathrm{r}^{\circ}-139 \mathrm{v}^{\circ}$ ).

96 Lacave 1971, 215-223 505-507, 516-517, 519-523, 618-619, 682. Thuaudet 2015, 143-145.

97 Thuaudet 2015, 147-149, fig. 16.

98 Blancard 1884-1885, to 2, doc 526.

99 Blancard 1884-1885, to 2 , doc 531.

100 Blancard 1884-1885, to 2 , doc 579.

101 Baratier, Reynaud 1951, 110.

102 Baratier, Reynaud 1951, 161. 
semble-t-il de peu d'importance, même au plus fort de la guerre entre Venise et les Turcs ${ }^{103}$.

En Provence même, la circulation du cuivre ou de ses alliages n'a été que rarement mise en évidence par les dépouillements bibliographiques et archivistiques. Les mentions de cuivre dans les tarifs de péage sont, comme pour les autres métaux, disposées le long du Rhône, et dans la vallée de la Durance dans la continuité de l'axe Marseille-Pertuis (fig. 6).

Le zinc, sous forme de calamine, est totalement absent des tarifs de péage, et il n'en est pas fait allusion dans les archives notariales hors des boutiques d'apothicaires, ce qui montre que son commerce n'a certainement jamais été très actif en Provence. Les tarifs médiévaux des péages provençaux mentionnent régulièrement le laiton, parfois sous forme d'objet finis ${ }^{104}$. Le laiton est nécessairement un produit d'importation, si on excepte la part de recyclage. Le commerce en Provence d'alliages en provenance du bassin de la Meuse, des contreforts des Ardennes et du massif de l'Eifel ${ }^{105}$ est fort probable, d'autant plus si on se reporte à la carte des attestations du mot laiton dans les tarifs de péages (fig. 6) : Il est intéressant d'observer que le laiton est absent des tarifs des Préalpes. Ces importations suffisaientelles à répondre aux besoins des artisans provençaux ? La documentation avignonnaise témoigne de l'existence d'une importante métallurgie du laiton en Lombardie durant la seconde moitié du XIV ${ }^{\text {e }}$ siècle ${ }^{106}$, mais cette industrie eut assurément une durée de vie bien plus longue. L'exploitation de gisements de calamine à peu de distance paraît donc nécessaire. L'exportation du laiton vers la Provence pourrait avoir été réalisée à destination du seul port de Marseille, puisque les tarifs de Toulon et Arles n'en font pas mention ${ }^{107}$. En 1320, treize balles de laiton sont transportées par cabotage de Marseille à Aigues-Mortes ${ }^{108}$.

Les seules mentions de bronze non ouvré dans les tarifs de péage (fig. 6) concernent celui du pont de Trinquetaille, à Arles ${ }^{109}$. Elles ne peuvent cependant constituer la preuve d'un commerce particulier de cet alliage avec le Roussillon. Il apparaît d'une part que le mot bronze et ses différentes traductions sont rares dans les textes. Mais surtout, une étude comparative semble révéler que le terme metallum recouvre souvent celui de bronze (fig. 7) ${ }^{110}$. On voit à travers cette

\footnotetext{
103 Collier, Billioud 1951, 516, 517, 549.

104 Thuaudet 2015, 147-149.

105 Day 1998, 133-134; Suttor 2014 ; Thomas et al. 2014.

106 Thuaudet 2015, 147-149, fig. 16.

107 Thuaudet 2015, 147-149, fig. 16.

108 Baratier, Reynaud 1951, 287.

109 Thuaudet 2015, fig. 16.

110 Thuaudet 2015, fig. 16.
}

nouvelle carte, que le nombre d'attestations augmente alors fortement.

Le cuivre brut se retrouve dans les textes sous la dénomination cuprum, en latin, courre en provençal. Allié à du zinc, le laiton est nommé lotonum, eletro sive lotono en latin, aurical et loton en provençal et ancien français. Avec de l'étain, il prend le nom d'aranum et d'erain. Les tarifs avignonnais identifient le cuivre sous forme de grenaille (granailla) ou de lingot en forme de pain (en pan) ou de plaque (en plata), de rosette ${ }^{111}$. La récupération de vieux cuivre en Provence est attestée par quelques actes isolés et par les tarifs de péage d'Avignon. Ils ne traduisent certainement pas l'ampleur du recyclage ${ }^{112}$.

\section{Les acteurs du commerce}

En Provence, à l'exception de quelques rares cas de particuliers, tel un notaire qui cède du cuivre à un peyrolier de Roaix en $1472^{113}$, la vente du métal non mis en forme est avant tout l'œuvre d'artisans ou de marchands pour lesquels elle n'est pas l'unique activité. Ainsi en est-il, par exemple, d'Étienne de Manduel ou de la filiale avignonnaise de la compagnie Datini qui trafiquent de tous types de marchandises. Le premier remet de l'étain en commande pour l'exportation en 1238, les seconds vendent à la fin du $\mathrm{XIV}^{\mathrm{e}}$ siècle de l'étain ${ }^{114}$ et envisagent le transport de cuivre ${ }^{115}$. En 1534, Claude Bernard, marchand d'Avignon est également revendeur de cuivre ${ }^{116}$. La même constatation est valable pour les nombreux fournisseurs de la cour papale d'Avignon dans le troisième tiers du XIV ${ }^{e}$ siècle. La fourniture d'étain et de plomb, parmi de multiples autres produits, est principalement le fait de marchands d'Avignon - cuivre et étain par Francisco Barralhi ${ }^{117}$, plomb et étain par le ferratier Guillaume Rostagni ${ }^{118}$, plomb et étain par Guillaume Barloyni ${ }^{119}$ - ou, dans le cadre des besoins de la Cour pontificale, d'agents de la papauté qui profitent par opportunisme de la nature de leurs fonctions pour faire venir du plomb à Avignon depuis Montpellier ${ }^{120}$, la Sardaigne ${ }^{121}$ ou Chypre ${ }^{122}$. La distribution

\footnotetext{
111 Lacave 1971, 205, 515, 520 ; Thuaudet 2015, fig. 16.

112 Lacave 1971, 520 ; Thuaudet 2015, fig. 16.

113 Lacave 1971, 216.

114 Antonietti 2007, 172-175, 789-798.

115 Antonietti 2007, $\mathrm{t}^{\circ} 2,194$.

116 Lacave 1971, 219.

117 Schäfer 1914, 251.

118 Schäfer 1914, 379.

119 Schäfer 1914, 503.

120 Schäfer $1914,314,504$ et 540.

121 Schäfer 1937, 15.

122 Schäfer 1937, 48.
} 


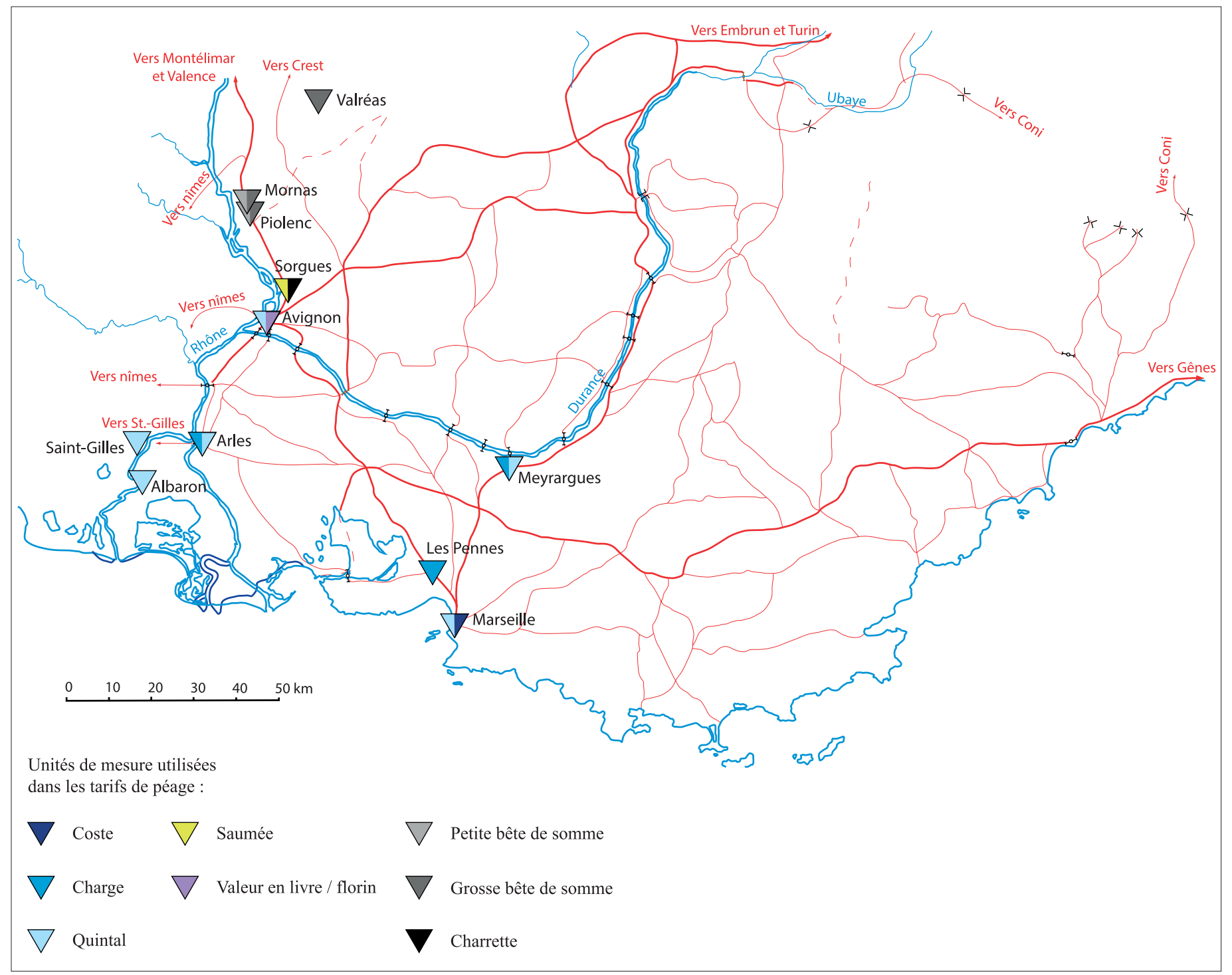

Fig. 7 - Carte des péages provençaux mentionnant le terme « métal ».

du métal auprès des fabricants est assurée par les marchands eux-mêmes, tels l'Aixois Guillaume d'Aquiné en $1421^{123}$ et le marseillais Raphaël Castagne en $1441^{124}$. Elle est également accomplie par des artisans jouant le rôle de revendeur : le potier d'étain aixois Raphaël Monterausi distribue du metallum, très certainement de l'étain, en 1423 et $1447^{125}$; le peyrollier marseillais Bérenger Cambalade fournit du metallum au campanier Jacques Bertrand ${ }^{126}$; le peyrolier et potier d'étain d'Avignon Jean Carrayron reconnaît en 1470 être débiteur de 109 florins à Jacques Bertrand, campanier d'Aix, probablement pour du métal ${ }^{127}$. Les martinerius vauclusiens ${ }^{128}$ et varois ${ }^{129}$ servent également probablement de relais entre les marchands et les artisans. Quelques-uns d'entre eux sont des peyroliers : il existe en effet une proximité de travail entre la production de demi-produits sous forme de tôles et la chaudronnerie ${ }^{130}$. Les réseaux de redistribution de la matière métallique ne sont donc pas exclusivement centrés sur les grandes villes de Provence.

\footnotetext{
123 AD BDR Aix, 309 E 116, fo $260 \mathrm{r}^{\circ}-260 \mathrm{v}^{\circ}, 1421$.

124 Reynaud 1956, 663.

125 AD BDR Aix, 307 E 12, n.f., 1423 ; 307 E 82, fo 176 vº 1447.

126 AD BDR Marseille, $391 \mathrm{E} 60, \mathrm{f}^{\circ} 275 \mathrm{~V}^{\circ}-277 \mathrm{r}^{\circ}$, 1461. Composition finale en 1462 : 308 E $558, f^{\circ} 146 r^{\circ}-147 r^{\circ}$.
}

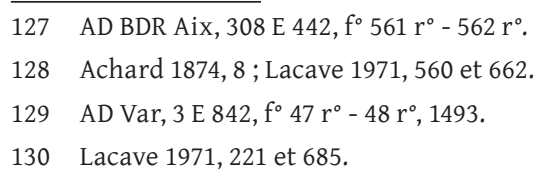




\section{Conclusion}

Les cartes de distribution des attestations des différents métaux dans les tarifs de péage, et la documentation d'archive, illustrent le rôle de colonne vertébrale tenu par la vallée du Rhône - qui passe par l'important marché de Lyon - et les parties médianes et hautes de la vallée de la Durance, prolongées par l'axe Marseille-Pertuis. Ces voies principales concentrent la circulation des métaux à l'état brut ou sous forme de demi-produits et assurent par l'intermédiaire d'un fin réseau secondaire, l'approvisionnement des régions plus éloignées. Cependant, l'embouchure du Rhône n'est pas, tout du moins à la fin du XVI ${ }^{e}$ siècle et au début du siècle suivant une voie de passage pour les métaux hormis le plomb, sans doute pour un usage local. Les cols de l'Ubaye sont un chemin d'accès pour les matériaux italiens ou du Dauphiné. Les échanges entre les deux voies principales se font notamment par une route joignant Marseille à Avignon, les deux principaux pôles économiques de Provence. La nature et l'intensité des échanges entre les cités de la rive gauche du Rhône et les territoires de la rive droite sont en revanche difficilement perceptibles. Marseille est la destination principale du transport maritime des métaux d'origine italienne, roussillonnaise, languedocienne ou pyrénéenne, surtout à partir du milieu du XIV e siècle où le poids économique d'Arles diminue de façon importante pour ne s'inverser réellement qu'à la fin du XVI e siècle. Marseille est également le point de départ majeur pour l'exportation de métaux vers les régions riveraines de la Méditerranée.

Le cheminement ouest-est au départ d'Avignon, passant par Aix et Nice, pour aboutir en Italie, est semble-t-il d'une moindre importance pour le commerce des métaux: les relations commerciales entre Nice, Toulon et Marseille apparaissent, d'une manière générale, assez restreintes, ce que semble confirmer le faible nombre de péages repérés ${ }^{131}$.

L'étude de la documentation écrite et archéologique illustre la dépendance des artisans provençaux vis-à-vis des approvisionnements alpins, dans une moindre proportion du midi de la France, voire d'origine beaucoup plus lointaine pour l'étain, la calamine, sous forme d'alliages, et le cuivre. Lactivité minière dans le cadre géographique de la Provence, à l'exception toutefois du plomb, mais il apparaît souvent de mauvaise qualité, est en effet très faible même si des prospections sont menées à plusieurs reprises. Le commerce des métaux n'est pas le fait de marchands spécialisés et des artisans le trafiquent parfois de façon opportuniste. Les informations manquent pour juger de la régularité des approvisionnements.

131 Une carte les répertoriant est disponible dans Baratier et al. 1969, carte 86.
La réalisation d'analyses de composition sur du matériel archéologique identifié comme ayant été produit ou ayant pu l'être en Provence permettrait peut-être de compléter, tout en tenant compte du recyclage des vieux métaux, la réflexion sur l'origine de l'approvisionnement en métaux et alliages. Pour ce faire, il est nécessaire de développer les études typologiques de mobilier métallique provençal, telle que celle que nous avons réalisée sur les accessoires du costume ${ }^{132}$. Il est également essentiel de multiplier les analyses de composition : le corpus de 155 objets du castrum Saint-Jean à Rougiers dans le Var que nous avons soumis à l'analyse est trop restreint et localisé pour fournir des éléments de réponse ${ }^{133}$. Une telle approche, de longue haleine, paraît être un des principaux moyens de progresser sur la compréhension de l'approvisionnement de la Provence en matières métalliques.

\section{Sources imprimées}

Becquet 1829 : L. Becquey, Mines et minières métalliques abandonnées ou qui n'ont point encore été exploitées en France, in : $\mathrm{T}$. Ravinet, Code des ponts et chaussées et des mines ou collection complète des lois, arrêtés, décrets, ordonnances, règlements et circulaires concernant le service des ponts et chaussées et des mines, jusqu'au $1^{\text {er }}$ janvier 1829, t. 3, Paris, Carilian-Cœury, 1829, 545 p., 200-218.

Blancard 1868 : L. Blancard, Essai surles monnaies de Charles Ir comte de Provence, Paris, J.-R. Dumoulin, 1868, 556 p.

Blancard 1884-1885 : L. Blancard, Documents inédits sur le commerce de Marseille édités intégralement ou analysés, Marseille, BarlatierFeissat père et fils, 2 t., 1884-1885, 417 p., 604 p.

Bonnaire 1802 : Bonnaire, 1802, Statistique du département des Hautes-Alpes, Paris, Imprimerie des sourds-muets, 1802, 113 p.

Bondurand 1901 : É. Bondurand, La leude et les péages de SaintGilles au XII ${ }^{e}$ siècle, textes en langue d'oc et en latin, Mémoires de l'Académie de Nîmes, VII série, XXIV, 1901, 267-292.

Darluc 1784 : M. Darluc, Histoire naturelle de la Provence, t. 2, Avignon, Jean Mossy, 1784, $315 \mathrm{p}$.

Féraud 1861 : J.-J.-M. Féraud, Histoire, géographie et statistique du département des Basses-Alpes, Digne, Vial, 1861, 744 p.

Roux 1862 : J. Roux, Statistique des Alpes-Maritimes, t. 1, Nice, Ch. Cauvin, 1862, 583 p.

Schäfer 1914 : K. H. Schäfer, Die Ausgaben der Apostolischen kammer unter Benedikt XII, Klemens V und Innocenz VI (1335-1362), Paderborn, Ferdinand Schöningh (Vatikanische Quellen zur Geschichte der Päpstlichen hof und Finanzverwaltung, 1316-1378, 3), 1914, 935 p.

Schäfer 1937 : K. H. Schäfer, Die Ausgaben der Apostolischen Kammer unter den päpsten Urban V und Gregor XI (1362-1378), Paderborn, Ferdinand Schöningh (Vatikanische Quellen zur Geschichte der Päpstlichen hof und Finanzverwaltung, 1316-1378, 6), 1937, 880 p. $\begin{array}{ll}132 & \text { Thuaudet } 2015 . \\ 133 & \text { Thuaudet } 2015 \text {, annexe } 2 .\end{array}$ 


\section{Références bibliographiques}

Achard 1874 : P. Achard, Notice sur la création, les développements et la décadence des manufactures de soie à Avignon, Mémoires de la société littéraire, scientifique et artistique d'Apt, t. 1, n 1 , feuille 2, $1874,67 \mathrm{p}$.

Ancel 1998 : B. Ancel, Techniques minières et maîtrise de l'espace dans les mines d'argent médiévales. Exemples de mines de plomb argentifère des Alpes du Sud (X ${ }^{\mathrm{e}} \mathrm{XIV}^{\mathrm{e}}$ siècles), in : P. Beck (dir.), L'innovation technique au Moyen Âge: actes du VIe Congrès international d'archéologie médiévale, Paris, Errance, 1998, 317 p., 108-110.

Ancel 2006a : B. Ancel, La mine d'argent du Fournel à L'ArgentièreLa-Bessée (Hautes-Alpes) : méthodologie et bilan, 1991-2001, in : $\mathrm{H}$. Barge (dir.), 4000 ans d'histoire des mines: l'exemple de la région Provence-Alpes-Côte d'Azur. Mélanges Paul Jacob, Theix, Actilia multimédia, 2006, 71-85, (Ouvrage sur Cd-Rom).

Ancel 2006b : B. Ancel, Archéologie minière dans les Alpes du Sud et en Provence, in: H. Barge (dir.), 4000 ans d'histoire des mines: l'exemple de la région Provence-Alpes-Côte d'Azur. Mélanges Paul Jacob, Theix, Actilia multimédia, 2006, 159-176 (Ouvrage sur Cd-Rom).

Ancel 2010 : B. Ancel, Les anciennes mines métalliques des Alpes du Sud : bilan diachronique, in : S. Tzortzis, X. Delestre (éd.), Archéologie de la montagne européenne, Paris, Errance, 2010, 333 p., 285-292.

Antonietti 2007 : F. Antonietti, Rapports commerciaux entre les compagnies Datini et Benini à la fin du XIV ${ }^{e}$ siècle (Arles-Avignon), thèse de doctorat d'histoire, Toulouse, Université de Toulouse le Mirail, 2007, 909 p.

Bailly-Maître 1989 : M.-C. Bailly-Maître, Pour une histoire des mines au Moyen Âge, l'exemple des mines de la terre d'Hierle (Gard), Archéologie du Midi médiéval, VII, 1989, 61-71.

Bailly-Maître 2000 : M.-C. Bailly-Maître, Essai d'une mine de plomb et argent versus arenas en Oisans par ordre du gouverneur du Dauphiné le 15 octobre 1359, in : A. Belmont (dir.), Pierres de mémoire, écrits d'histoire: pages d'histoire en Dauphiné offertes à Vital Chomel, Grenoble, Presses Universitaires de Grenoble, (La Pierre et l'Ecrit), 2000, 451 p., 131-143.

Bailly-Maître 2002 : M.-C. Bailly-Maître, Les carreaux miniers d'une grande exploitation médiévale en Languedoc ( $\mathrm{XI}^{\mathrm{e}}-\mathrm{XIV}^{\mathrm{e}}$ siècles) ", Archéologie en Languedoc, 26, 2002, 97-112.

Bailly-Maître 2007 : M.-C. Bailly-Maître, Pouvoir et mines d'argent au Moyen Âge, mainmise politique et évidences archéologiques, in : M.-C. Bailly-Maître et J.-M. Poisson (dir.), Mines et pouvoir au Moyen Âge, Lyon, Presses universitaires de Lyon, (Collection d'histoire et d'archéologie médiévales, 20), 2002, 213 p., 21-46.

Bailly-Maître 2010 : M.-C. Bailly-Maître, Saint-Laurent-le-Minier, Pommiers, Saint-Bresson, Montdardier (Gard), District minier médiéval d'Hierle, Archéologie médiévale (Chronique des fouilles), 40, 2010, 324-325.

Bailly-Maître 2012 : M.-C. Bailly-Maître, La Terre d'Hierle et ses mines au Moyen Âge, De roches et d'Hommes: mines et minéraux en Cévennes, Le Vigan, Musée Cévenol, 2012, 5-19.

Bailly-Maître, Dhénin 2004 : M.-C. Bailly-Maître, M. Dhénin, Ateliers monétaires et mines d'argent dans les Alpes ( $\mathrm{X}^{\mathrm{e}}-\mathrm{XV}^{\mathrm{e}}$ siècles) : histoire, archéologie, numismatique et archéométrie », Archéologie médiévale, 34, 2004, p. 43-64.

Bailly-Maître, Dupraz 1994 : M.-C. Bailly-Maître, J. Dupraz, Brandes en Oisans. La mine d'argent des Dauphins (XII-XIV ${ }^{e}$ s.). Isère, Lyon, ALPARA, (Documents d'archéologie en Rhône-Alpes et en Auvergne, 9), 1994, 169 p.

Baratier, Reynaud 1951 : É. Baratier, F. Reynaud, Histoire du commerce de Marseille, t. II, De 1291 à 1480, Paris, Plon, 1951, 922 p.

Baratier, Ruby, Hildesheimer 1969 : É. Baratier, G. Ruby, E. Hildesheimer, Atlas historique: Provence, Comtat, Orange, Nice, Monaco, Paris, Librairie Armand Colin, 1969, 220 p.

Barge 2006 : H. Barge, Les minéralisations cuprifères et leurs exploitations (-2500 av. J.-C./ XX $\mathrm{XX}^{\mathrm{e}}$ siècle) dans le Sud-Est de la France, état des recherches pour la préhistoire, in : H. Barge (dir.), 4000 ans d'histoire des mines : l'exemple de la région Provence-Alpes-Côte d'Azur. Mélanges Paul Jacob, Theix, Actilia multimédia, 2006, 11-25, (Ouvrage sur Cd-Rom).

Benoit 1960 : F. Benoit, L'économie de la Provence à l'époque antique, II, Le grenat des marseillais et les mines des Maures, Revue d'études ligures, XXVI ${ }^{\mathrm{e}}$ année, 1960, 221-232.

Benoit, Braunstein 1983 : P. Benoit, P. Braunstein, Les comptes miniers d'Hurtières en Savoie (1338-1350), in : P. Benoit et P. Braunstein (dir.), Mines, carrières et métallurgie dans la France médiévale, Paris, Edition du CNRS, 1983, 415 p., 183-206.

Bergasse 1954 : L. Bergasse, De 1599 à 1660, in : L. Bergasse, G. Rambert (dir.), Histoire du commerce de Marseille, IV, Paris, Plon, $683 \mathrm{p}$.

Bernardi 1990 : P. Bernardi, Métiers du bâtiment et techniques de construction à Aix-en-Provence à la fin de l'époque gothique (1400-1500), thèse de 3e cycle, Aix-en-Provence, Université d'Aix-Marseille I, 1990, 355 p., 734 p.

Berthet 2007 : M.-P. Berthet, Pouvoir et industrie minière en Provence, in : M.-C. Bailly-Maître et J.-M. Poisson (dir.), Mines et pouvoir au Moyen Âge, Lyon, Presses universitaires de Lyon, (Collection d'histoire et d'archéologie médiévales, 20), 2002, 213 p., 63-76.

Billioud 1958 : J. Billioud, Les mines de plomb des Basses-Alpes (XVI ${ }^{e}$-XIX ${ }^{e}$ siècle) », Provence historique, VIII, fasc. 31, 1958, 42-52.

Bois 2000 : G. Bois, La grande dépression médiévale: $X I V^{e}-X V^{e}$ siècles, le précédent d'une crise systémique, Paris, Presses universitaires de France, 2000, $211 \mathrm{p}$.

Braunstein 2016 : P. Braunstein, Les Allemands à Venise (1380-1520), Rome, Ecole Française de Rome, (Bibliothèque des Écoles françaises d'Athènes et de Rome, 372), 2016, 975 p.

Casini 2007 : A. Casini, L'extraction du cuivre et de l'argent dans les Monts de Campiglia Marittima (Toscane) de l'époque étrusque au XVI ${ }^{\text {e }}$., in: M.-C. Bailly-Maître et J.-M. Poisson (dir.), Mines et pouvoir au Moyen Âge, Lyon, Presses universitaires de Lyon, (Collection d'histoire et d'archéologie médiévales, 20), 2002, 213 p., 103-111.

Chapelot 1985 : O. Chapelot, Les ouvriers du métal en Bourgogne à la fin du Moyen Âge : l'exemple du Châtillonais, in : O. Chapelot et P. Benoit (dir.), Pierre et métal dans le bâtiment au Moyen Âge, Paris, EHESS, 1985, 370 p., 305-318. 
Collier, Billioud 1951 : R. Collier, J. Billioud, Histoire du commerce de Marseille, t. III, De 1480 à 1599, Paris, Plon, 1951, 563 p.

Coulet 1975 : N. Coulet, Prospections minières en Provence à la fin du Moyen Âge, in : Mines et métallurgie, XII ${ }^{e}-X V I^{e}$ siècle : actes du $98^{e}$ Congrès national des sociétés savantes, Paris, BNF, 1975, 173 p., 159-168.

Crabières 2001 : L. Crabières, L'exploitation du minerai des Hurtières en Savoie au XVII ${ }^{\mathrm{e}}$ siècle, in : M.-C. Bailly-Maître, A. Ploquin, N. Garioud (dir.), Le fer dans les Alpes du Moyen-Âge au $X I X^{e}$ siècle : actes du colloque international de Saint-Georges-d'Hurtières, Montagnac, Monique Mergoil, 2001, 243 p., 59-69.

Day 1990 [1998] : J. Day, Brass and zinc in Europe from the middle ages until the mid-nineteenth century, in: P. T. Craddock (dir.), 2000 years of zinc and brass, (révisé), British Museum, occasional paper, 50, Londres, British Museum, 1990 [1998], 258 p., 133-156.

Di Gangi 2001 : G. Di Gangi, L'attività estrattiva e metallurgica nel Piemonte medievale : spostamenti di maestranze e trasmissione di tecnologie « imprenditori minerari » ed insediamenti specializzati, in : P. Braunstein (dir.), La sidérurgie alpine en Italie (XII ${ }^{-}$XVII ${ }^{e}$ siècle), Rome, École française de Rome, (Collection de l'Ecole française de Rome, 290), 2001, 624 p., 327-392.

Di Gangi 2007 : G. Di Gangi, Ressources minières et centres de pouvoir en Piémont médiéval, in : M.-C. Bailly-Maître et J.-M. Poisson (dir.), Mines et pouvoir au Moyen Âge, Lyon, Presses universitaires de Lyon, (Collection d'histoire et d'archéologie médiévales, 20), 2002, 213 p., 77-102.

Février 1964 : P.-A. Février, Le développement urbain en Provence, de l'époque romaine à la fin du XIV ${ }^{e}$ siècle (archéologie et histoire urbaine), Paris, E. de Boccard, 1964, 231 p.

Garioud 2007 : N. Garioud, La maison de Savoie et ses mines d'argent, essai d'analyse générale d'une politique minière (fin $\mathrm{XIII}^{\mathrm{e}}$ - première moitié XVI ${ }^{\mathrm{e}}$ ), in : M.-C. Bailly-Maître et J.-M. Poisson (dir.), Mines et pouvoir au Moyen Âge, Lyon, Presses universitaires de Lyon, (Collection d'histoire et d'archéologie médiévales, 20), 2002, 213 p., 47-61.

Guillot, Benoit 1994 : I. Guillot, P. Benoît, Extraction de l'argent des minerais de cuivre au XVI ${ }^{\mathrm{e}}$ siècle : le livre XI du De Metallica d'Agricola, in: P. Benoît (dir.), Mines et métallurgie, s.l., Programme Rhône-Alpes, Recherches en sciences humaines, 1994, 310 p., 107-133.

Hatcher 1973 : J. Hatcher, English tin production and trade before 1550, Oxford, Clarendon Press, 1973, XI-219 p.

Lacave 1971 : M. Lacave, Entreprises industrielles comtadines (14601560), thèse de $3^{\mathrm{e}}$ cycle, Montpellier, Université de Montpellier, 1971, $755 \mathrm{p}$.

Lanza 1997 : M.-P. Lanza, Archéologie minière dans le Massif des Maures (Var) : prospection thématique portant sur les mines et les sites métallurgiques du Massif des Maures, approche diachronique, mémoire de DEA, Aix-en-Provence, Université de Provence, 1997, 229 p.

Lanza-Berthet 2006 : M.-P. Lanza-Berthet, Synthèse des recherches sur les mines et la métallurgie dans le massif des Maures (19952001), in : H. Barge (dir.), 4000 ans d'histoire des mines: l'exemple de la région Provence-Alpes-Côte d'Azur. Mélanges Paul Jacob, Theix, Actilia multimédia, 2006, 149-158, (Ouvrage sur Cd-Rom).
Léchelon 1997 : B. Léchelon, La mine d'argent médiévale de BoucoPayrol (Aveyron), in : Archéologie en Languedoc: actes du colloque Mines et métallurgie en Languedoc-Roussillon de la Préhistoire au Moyen Âge, Cabrières, Fédération archéologique de l'Hérault, (Archéologie en Languedoc, 21), 1997, 245 p., 167-182.

Lopez 1989 : M. Lopez, Observation sur les techniques minières antiques et médiévales: quelques exemples dans la région de Lodève (Hérault), in: Mines et métallurgies antiques et médiévales de la France méridionale, recherches récentes: actes des Journées de Perpignan, Perpignan, Ville de Perpignan, 1989, 134 p., 111-121.

Maas 2002 : Maas A., 2002, La mine de La Roussignole 2 (Cabrières, Hérault): Premiers résultats des recherches, Archéologie en Languedoc, 26, 2002, 55-62.

Malham 2010 : A. Malham, The classification and interpretation of tin smelting remains from South west England: a study of the microstructure and chemical composition of tin smelting slags from Devon and Cornwall, and the effect of technological developments upon the character of slags, thèse de $3^{\text {e }}$ cycle, Bradford, Université de Bradford, 2010, 686 p.

Mari 1979 : G. Mari, Mines et minéraux de la Provence cristalline: Maures, Estérel, Tanneron, Nice, Serre, 1979, 254 p.

Meyer, Thomas, Wyss 2014 : O. Meyer, N. Thomas, M. Wyss, Témoins archéologiques d'un atelier de bronzier travaillant à Saint-Denis à la fin du XVI e siècle, in : D. Bourgarit, J. Bassett, F. Bewer (dir.), French bronze sculpture: materials and techniques, 16th - 18th century, Londres, Archetype Publications, 2014, 249 p., 215-224.

Morin, Guiomar 2006 : D. Morin, M. Guiomar, L'exploitation du minerai de plomb argentifère dans les Alpes-de-Haute-Provence (XIV ${ }^{\mathrm{e}}$-XVIII ${ }^{\mathrm{e}}$ siècles) », in : H. Barge (dir.), 4000 ans d'histoire des mines: l'exemple de la région Provence-Alpes-Côte d'Azur. Mélanges Paul Jacob, Theix, Actilia multimédia, 2006, 87-110, (Ouvrage sur Cd-Rom).

Papon 1780 : P. Papon, Voyage littéraire de Provence, Paris, Barrois l'aîné, 1780, 457 p.

Payn-Échalier 2006 : P. Payn-Échalier, Les activités portuaires d'Arles à la fin du XVI ${ }^{\mathrm{e}}$ siècle, Provence Historique, LVI, fasc. 224, 2006, 195-221.

Pernoud 1951 : R. Pernoud, Le Moyen Âge jusqu'en 1291, in : Histoire du commerce de Marseille, t. I, Antiquité, Moyen Âge jusqu'en 1291, Paris, Plon, 1951, 384 p., 109-376.

Poly 1976 : J.-P. Poly, La Provence et la société féodale (979-1166). Contribution à l'étude des structures dites féodales dans le Midi, Paris, Bordas, $431 \mathrm{p}$.

Reynaud 1956 : F. Reynaud, Le mouvement des navires et de marchandises à Port-de-Bouc à la fin du XV $\mathrm{XV}^{\mathrm{e}}$ siècle, Revue d'histoire économique et sociale, vol. XXXIV, n² 2, 1956, 153-170.

Schick 1957 : L. Schick, Un grand homme d'affaires au début du XVI $I^{e}$ siècle, Jacob Fugger, Paris, S.E.V.P.E.N., 1957, 323 p.

Sclafert 1926 : T. Sclafert, Le Haut-Dauphiné au Moyen Âge, Paris, Société anonyme du recueil Sirey, 765 p.

Serra 1992 : F. Serra, La métallurgie du plomb sur le domaine d'Orves (Évenos, Var), mémoire de Maîtrise d'Archéologie médiévale, Aixen-Provence, Université d'Aix-Marseille I, 1992, 122 p.

Suttor 2014 : M. Suttor, La dinanderie, fille de la Meuse, in : N. Thomas, I. Leroy, J. Plumier (dir.), L'or des dinandiers, fondeurs et batteurs mosans 
au Moyen Âge, Bouvignes, Dinant, Maison du patrimoine médiéval mosan (Cahiers de la MPMM, 7), 2014, 132 p., 21-30.

Thuaudet 2015 : 0 . Thuaudet, Les accessoires métalliques du vêtement et de la parure de corps en Provence du XI $I^{e}$ au XVI siècle: étude archéologique et approche croisée d'une production méconnue, thèse de doctorat d'archéologie, Aix-en-Provence, Aix-Marseille Université - LA3M, 5 vol., 1402 p., 626 fig., 40 fig.

Thomas et al. 2014 : Thomas N., Leroy I., Plumier J. (dir.), L'or des dinandiers, fondeurs et batteurs mosans au Moyen Âge, Bouvignes,
Dinant, Maison du patrimoine médiéval mosan (Cahiers de la MPMM, n 7), 2014, 135 p.

Verna 2017 : C. Verna, L'industrie au village. Essai de micro-histoire (Arles-sur-Tech, XIV et XV ${ }^{e}$ siècles), Paris, Les Belles Lettres, 2017, $552 \mathrm{p}$.

Weiberger 1990 : S. Weiberger, La transformation de la société paysanne en Provence médiévale, Annales. Économies, Sociétés, Civilisations, $45^{\text {ème }}$ année, nº 1, 1990, 3-19. 
Arles Adrien

Arkemine SARL

Adrien.arles@arkemine.fr

\section{Bailly-Maître Marie-Christine}

Aix Marseille Univ, CNRS, LA3M, Aix-en-Provence, France baillymaitre@wanadoo.fr

\section{Benvenuti Marco}

Università di Firenze, Dipartimento Scienze della Terra

m.benvenuti@unifi.it

\section{Bianchi Giovanna}

Università di Siena, Dipartimento di Scienze Storiche e dei Beni Culturali

giovanna.bianchi@unisi.it

\section{Boisseuil Didier}

Université de Tours, Cethis, EA 6298

didier.boisseuil@wanadoo.fr

\section{Bonnamour Gérald}

Arkemine SARL, association EESV, membre associé du laboratoire Traces (UMR 5608, Toulouse)

gerald.bonnamour@arkemine.fr

\section{Bonnamour Romain}

association EESV (Equipe d'Exploration Spéléologique de Villefranche)

rb@rb-ebeniste.fr

\section{Braunstein Philippe}

École des Hautes Études en Sciences Sociales

philippe_braunstein@yahoo.fr

\section{Bresc Cécile}

Université Paris IV Sorbonne, Orient et Méditerranée, UMR 8167

ratepenade@yahoo.fr

\section{Capel Chloé}

UMR 8167 - Orient et Méditerranée, équipe Islam médiéval chloe.capel@gmail.com

\section{Casagrande Lara}

Ecomuseo Argentario

info@ecoargentario.it

\section{Chiarantini Laura}

Università di Firenze, Dipartimento Scienze della Terra

laura.chiarantini@unifi.it

\section{Cicali Cristina}

Università di Siena, Dipartimento di Scienze Storiche e dei Beni Culturali

cristinacicali@yahoo.it

\section{Claughton Peter}

University of Exeter

P.F.Claughton@exeter.ac.uk

\section{Condorelli Francesca}

Fondazione Bruno Kessler (FBK)

francesca.condorelli@hotmail.it

\section{Dallai Luisa}

Università di Siena, Dipartimento di Scienze Storiche e dei Beni Culturali

luisa.dallai@unisi.it

\section{Donati Alessandro}

Università di Siena, Dipartimento di Biotecnologie, Chimica e Farmacia

alessandro.donati@unisi.it

Fabijanec Sabine-Florence

Croatie Academy of Sciences ans Arts (CASA/HAZU)

flofaber@hazu.hr 


\section{Faucher Thomas}

UMR 5060 IRAMAT-Centre Ernest Babelon, CNRS, Université d'Orléans

thomas.faucher@cnrs-orleans.fr

\section{Flament Julien}

UMR 5060 IRAMAT-CEB, Université d'Orléans, 3D rue de la Férollerie, 45071 Orléans Cedex 2, France

julien.flamen@gmail.com

\section{Gattiglia Anna}

Università degli Studi di Torino, Dipartimento di Studi Storici anna.gattiglia@unito.it.

\section{Gauthier Joseph}

CNRS, Chrono-environnement, UMR 6249, Université de Haute-Alsace, CRESAT EA 3436

josep.gauthier@laposte.net

\section{Leleu Florian}

Arkemine SARL

florian.leleu@arkemine.fr

Marchand Julie

Université de Poitiers, HERMA, EA 3811

julie.mj.marchand@gmail.com

\section{Martinez Elcacho Albert}

Universitat de Lleida (UdL)

albert.martinez@historia.udl.cat

\section{Minvielle Larousse Nicolas}

Aix Marseille Univ, CNRS, LA3M, Aix-en-Provence, France minvielle.nicolas@gmail.com

\section{Montel Aurélien}

Université Lumière-Lyon 2 - CIHAM-UMR 5648

aurelien.montel@gmail.com

\section{Nefzaoui Souha}

Faculté des Sciences Humaines et Sociales de Tunis (9 Avril) (FSHST)

nefzaouis@yahoo.fr

\section{Neri Elisabetha}

Université Paris-Sorbonne, OM, UMR 8167

Elisabetta.neri@unicatt.it

\section{Patria Luca}

Centro Ricerche di Cultura Alpina, Torino

culturealpine@gmail.com

\section{Pezzica Ilaria}

Università degli Studi di Torino, Dipartimento di Studi Storici ilaria.pezzica@gmail.com

\section{Poisson Jean-Michel}

EHESS, CIHAM, UMR 5648

Jean-Michel.Poisson@univ-lyon2.fr

\section{Rabot Alexandre}

Université Lyon 2 Lumière, HiSoMA, UMR 5189

alexandre.rabot@mom.fr

\section{Redon Bérangère}

CNRS, HiSoMA, UMR 5189

berangere.redon@mom.fr

\section{Roat Giulia}

Università degli Studi di Padova

giulia.roat@gmail.com

\section{Rossi Maurizio}

Il Patrimonio Storico-Ambientale, Torino

presidenza@aipsam.org

\section{Rovelli Alessia}

Università della Tuscia, Dipartimento di Studi linguisticoletterari, storico-filosofici e giuridici

rovelli@unitus.it

\section{Sarah Guillaume \\ UMR 5060 IRAMAT-Centre Ernest Babelon, CNRS, Université d'Orléans \\ guillaume.sarah@cnrs-orleans.fr}

\section{Scoz Jacopo}

Università degli Studi di Siena

scoz.jacopo@gmail.com

\section{Straßburger Martin}

Ludwig-Maximilians-Universität München and Consultancy for Mining Archaeology

martin@miningarchaeology.com

\section{Téreygeol Florian}

LAPA-IRAMAT, NIMBE, CEA, CNRS, Université Paris-Saclay, CEA Saclay 91191 Gif-sur-Yvette France

florian.tereygeol@cea.fr

\section{Thaler Federico}

Università degli Studi di Trento

vukovarstoner@gmail.com 


\section{Thuaudet Olivier}

Aix Marseille Univ, CNRS, LA3M, Aix-en-Provence, France olivier.thuaudet@laposte.net

\section{Tomas Emilie}

Arkemine SARL

emilie.tomas@gmail.com

\section{Verna Catherine}

Université Paris 8, HISPOSS, EA 1571

catherine.verna@wanadoo.fr

\section{Villa Igor M.}

Institut für Geologie, Universität Bern; Centro Universitario Datazioni e Archeometria, Univ. Milano-Bicocca

igor@geo.unibe.ch

\section{Vingo (de) Paolo}

Università degli Studi di Torino, Dipartimento di Studi Storici paolo.devingo@unito.it

\section{Volpi Vanessa}

Università di Siena, Dipartimento di Biotecnologie, Chimica e Farmacia

vaniv@hotmail.it 


\section{Table des matières}

Remerciements

Xavier Delestre, Nicolas Faucherre

Préfaces

Nicolas Minvielle Larousse, Marie-Christine Bailly-Maître, Giovanna Bianchi

Avant-Propos

Marie-Christine Bailly-Maître

Introduction - Les métaux précieux en Méditerranée médiévale. Exploitations, transformations, diffusions.

État de la recherche

\section{LES ESPACES MINIERS MÉDITERRANÉENS}

Giovanna Bianchi, Luisa Dallai

Le district minier des Collines Métallifères (Toscane, Italie) durant la période médiévale.

L'exploitation des ressources et les implications politiques et économiques

Marco Benvenuti, Laura Chiarantini, Cristina Cicali, Igor M. Villa, Vanessa Volpi

La produzione d'argento nel distretto minerario di Montieri-Massa Marittima (Colline Metallifere, Toscana

meridionale). Alcune considerazioni su dati recenti

Anna Gattiglia, Ilaria Pezzica, Maurizio Rossi, Paolo de Vingo avec la collaboration de Luca Patria

Production et destination de l'argent du district minier des vallées de Lanzo (Turin) pendant la première moitié du XIV ${ }^{e}$ siècle

Lara Casagrande, Martin Straßburger, Francesca Condorelli, Giulia Roat, Federico Thaler, Jacopo Scoz

Medieval silver mining on the Monte Calisio plateau (Trentino - Italy)

Jean-Michel Poisson

L'entreprise pisane d'exploitation des mines d'argent de I'Iglesiente (Sardaigne), XIII'-XIVe siècles

Nicolas Minvielle Larousse

Les lieux et les rythmes de la production argentifère médiévale en Languedoc oriental

Florian Leleu avec la collaboration d'Adrien Arles et Emilie Tomas

Les mines de cuivre de Castifao 
Albert Martínez Elcacho

The silver mines of Falset (Catalonia).

Development, regulation and organization in mid-14th century

Peter Claughton

View from the other side of the Channel.

England's mining response to the silver crisis in the 15th century

Souha Nefzaoui

L'argent monétaire au haut Moyen Âge en Ifrīqiya. Une étude à mener

Julie Marchand, Thomas Faucher, Alexandre Rabot, Bérangère Redon, Florian Téreygeol

L'exploitation de l'or en Égypte au début de l'époque islamique. L'exemple de Samut

\section{TECHNIQUES ET ORGANISATION DE LA PRODUCTION}

Joseph Gauthier, Catherine Verna

Les prospections minières.

Des terrains et des princes (comté du Roussillon et duché de Bourgogne, $\mathrm{XV}^{\mathrm{e}}$ siècle)

Didier Boisseuil

Prospections minières et production de métaux précieux dans le sud de la Toscane à la fin du XVe siècle.

Quelques observations

Gérald Bonnamour, Romain Bonnamour

La mine médiévale et moderne de Cella à Joux en Beaujolais.

Un petit gisement d'argent de renom en marge des importantes exploitations minières Lyonnaises

Florian Téreygeol

La préparation des minerais argentifères au Moyen Âge.

Choix technique ou contrainte économique?

Julien Flament, Florian Téreygeol, Guillaume Sarah

La production du cuivre et de ses alliages à Castel-Minier (Ariège, France).

Opportunisme métallurgique et pragmatisme économique d'une fonderie de moyenne montagne

au XVe siècle

Vanessa Volpi, Luisa Dallai, Alessandro Donati

L'uso di tecnologie ad elevate prestazioni, pXRF, per lo studio del distretto minerario delle colline metallifere (Toscana, Italia)

\section{CIRCULATIONS ET USAGES DES MÉTAUX}

Aurélien Montel

D'Awdag̉ust à Cordoue. L'or du Soudan et la politique maghrébine du califat umayyade d'al-Andalus

(IVe/Xe siècles)

Chloé Capel

L'or africain et le paradoxe de Sijilmassa (Maroc - VIIIe-XIVe siècles).

Atelier de frappe primordial, histoire méconnue 
Ressources métalliques et frappes monétaires dans le Proche-Orient au temps des califats.

Le cas du Bilād al-Shām médiéval

Elisabetta Neri

Les sources de l'or du décor entre Orient et Occident (IV'e-XIle siècles).

Or monétaire, réutilisation, or frais

Guillaume Sarah

L'emploi du laiton dans les monnayages d'argent médiévaux.

État des connaissances actuelles et perspectives de recherche

Olivier Thuaudet

Approvisionnement et circulation du cuivre et de ses éléments d'alliage en Provence du XIII ${ }^{e}$ au XVle siècles. L'apport des sources écrites et archéologiques

Sabine Florence Fabijanec

Le circuit de l'argent de l'Adriatique orientale à Alexandrie à la fin du XIV ${ }^{e}$ siècle

Philippe Braunstein

Conclusion - Les métaux précieux en Méditerranée. Productions, transformations, circulations 


\title{
Les métaux précieux en Méditerranée médiévale
}

\author{
Exploitations, transformations, circulations
}

\section{ARCHÉOLOGIES MÉDITERRANÉENNES}

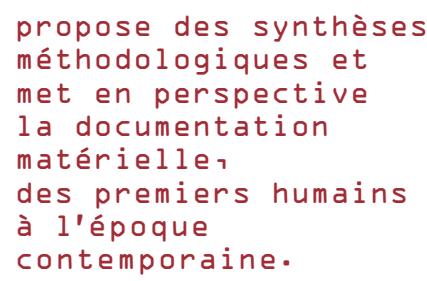

\section{BiAMA}

Dans la lignée des anciens Travaux du Centre Camille Jullian, la Bibliothèque d'archéologie méditerranéenne et africaine (BiAMA) propose des ouvrages relatifs à I'histoire et à l'archéologie de la Méditerranée antique et médiévale, en particulier de Marseille et de la Provence.

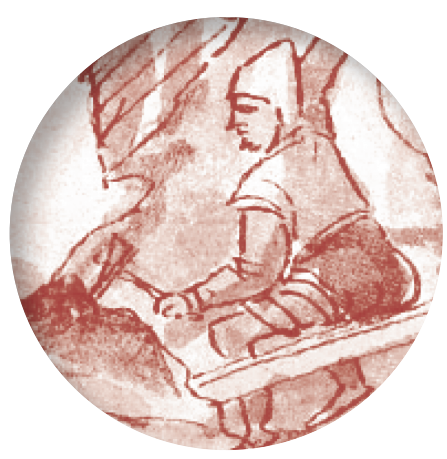

Illustration de $1^{\text {re }}$ et $4^{\mathrm{e}}$ de couverture Das Schwazer Bergbuch, 1556
Cet ouvrage rassemble les actes d'un colloque international tenu à la Maison méditerranéenne des sciences de I'homme d'Aix-en-Provence les 6, 7 et 8 octobre 2016. Organisée par le Laboratoire d'Archéologie Médiévale et Moderne en Méditerranée (CNRS, Aix-Marseille Université) et par le Dipartimento di Scienze Storiche e dei Beni Culturali de I'Université de Sienne, cette rencontre a réuni près de cinquante spécialistes du Moyen Âge - archéologues, historiens, géochimistes - autour des métaux précieux en Méditerranée.

Ce livre entend examiner un long processus qui s'étend de l'extraction des minerais jusqu'à la diffusion des métaux, en articulant les techniques aux sociétés et aux pouvoirs. Le panorama des principaux lieux de la production, ici présenté sous forme de synthèses régionales, côtoie des études qui suivent les différentes phases de ce processus, de façon à confronter les sources, les approches et à faciliter les comparaisons. L'enquête se focalise enfin sur les circulations des métaux, en mettant en évidence les problèmes de sources et de méthodes que cela induit, tout en proposant des relectures historiographiques fondées sur les résultats de travaux récents.

Pour la première fois, la Méditerranée médiévale fournit le cadre géographique d'une réflexion collective consacrée aux productions et circulations des métaux précieux. Au-delà du simple bilan de connaissances, ce livre entend finalement proposer quelques jalons pour une histoire comparée et connectée : l'approfondissement, puis la confrontation d'enquêtes monographiques et régionales offrent dans cette optique de fécondes perspectives pour les années à venir.

Nicolas Minvielle Larousse est chercheur post-doctorant d'Aix-Marseille Université. Marie-Christine Bailly-Maître est directrice de recherche émérite au CNRS.

Giovanna Bianchi est professeure d'archéologie médiévale à l'Université de Sienne.
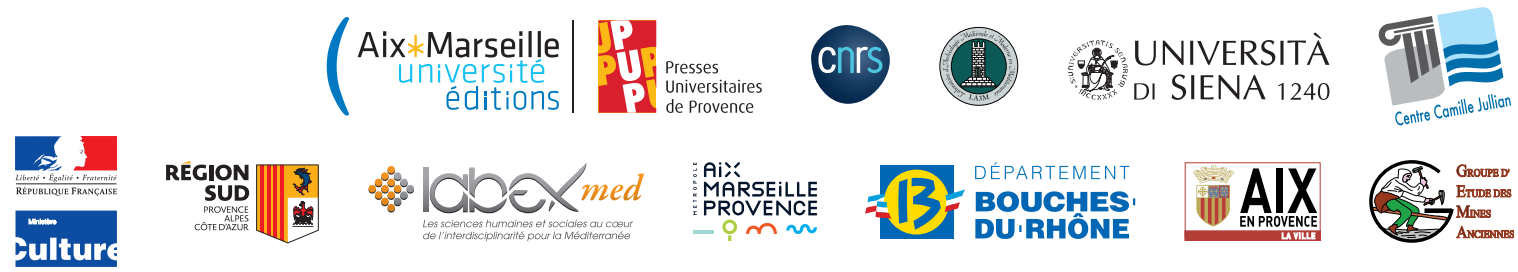

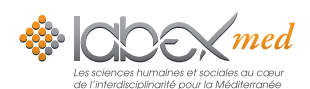

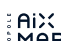
MARSELLE PROVENCE

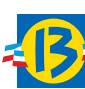

DÉPARTEMENT BOUCHES DU'RHÔNE
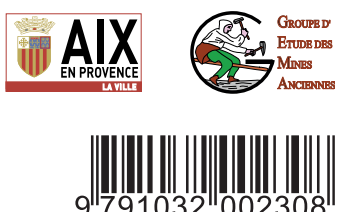